Lars Jenßen, Oliver Thiel, Simone Dunekacke \& Sigrid Blömeke

Titel (deutsch): Mathematikangst bei angehenden frühpädagogischen Fachkräften: Bedeutsam für professionelles Wissen und Wahrnehmung von mathematischen Inhalten im Kita-Alltag?

Titel (englisch): Early Childhood Teacher Students' Mathematics Anxiety: Relevant for Professional Knowledge and Perception of Mathematics in Preschool?

Dr. Lars Jenßen

Humboldt-Universität zu Berlin

Unter den Linden 6

10099 Berlin

E-Mail: lars.jenssen@hu-berlin.de

Telefon: 030/2093-66823

Prof. Dr. Oliver Thiel

Department of Mathematics, Queen Maud University College of Early Childhood Education, Trondheim

Thrond Nergaards veg 7

7044 Trondheim, Norwegen

Prof. Dr. Simone Dunekacke

Freie Universität Berlin

Habelschwerdter Allee 45

14195 Berlin

Prof. Dr. Sigrid Blömeke

Centre for Educational Measurement at the University of Oslo (CEMO)

Postboks 1161, Blindern

0318 Oslo, Norwegen 


\section{Mathematikangst bei angehenden frühpädagogischen Fachkräften: Bedeutsam für professionelles Wissen und Wahrnehmung von mathematischen Inhalten im Kita- Alltag?}

Zusammenfassung (deutsch):

Die Fertigkeit, mathematische Inhalte in Spielsituationen der Kindertagesstätte zu identifizieren, um diese für eine angemessene Förderung mathematischer Kompetenzen bei Kindern zu nutzen, kann als ein wesentlicher Bestandteil professioneller Kompetenz von frühpädagogischen Fachkräften verstanden werden. Diese mathematikbezogene Situationswahrnehmung sollte dabei vor dem Hintergrund von sowohl fachmathematischem als auch mathematikdidaktischem Wissen reflektiert werden. Inwieweit Emotionen an dem Prozess der Situationswahrnehmung beteiligt sind, wurde bisher nicht untersucht. Mathematikangst stellt eine bedeutsame Leistungsemotion bei (angehenden) frühpädagogischen Fachkräften dar. Der vorliegende Beitrag untersucht daher die Frage, inwieweit Mathematikangst und professionelles mathematikbezogenes Wissen die mathematikbezogene Situationswahrnehmung von angehenden frühpädagogischen Fachkräften vorhersagen. Hierfür wurden $n=354$ angehende frühpädagogische Fachkräfte befragt und mit paper-pencil-basierten und videobasierten Tests hinsichtlich ihrer professionellen Kompetenz getestet. Die Ergebnisse deuten unter anderem auf bedeutsame indirekte Effekte von Mathematikangst, vermittelt über das mathematische Fachwissen und das mathematikdidaktische Wissen, auf die mathematikbezogene Situationswahrnehmung hin. Die Relevanz der Ergebnisse wird vor dem Hintergrund der Ausbildung von frühpädagogischen Fachkräften diskutiert.

Schlüsselwörter: Frühpädagogische Fachkräfte, Mathematikangst, professionelles Wissen, Situationswahrnehmung,

Abstract (englisch):

The skill to perceive math-related situations during play-based activities in preschool can be conceptualized as an inherent part of early childhood teachers' professional competence. It is an important aspect for fostering children's mathematical competencies and should be based on mathematics content knowledge as well as mathematics pedagogical content knowledge. How emotions affect the perception of mathematics-related situations is still unknown. Mathematics anxiety can be seen as a relevant achievement emotion of (pre-service) early childhood teachers. The current study investigates the effects of mathematics anxiety, mathematics content knowledge and mathematical pedagogical content knowledge on early childhood teacher students' math-related perception. Therefore, $n=354$ early childhood teacher students were surveyed with well-established questionnaires and tested with paper-pencil-based as well as video-based assessments on their mathematics anxiety, knowledge and perception skills. Results indicate significant indirect effects of mathematics anxiety on math-related perception, mediated by knowledge. Our findings are discussed with respect to early childhood teachers' training in Germany.

Keywords: early childhood teachers, mathematics anxiety, professional knowledge, mathrelated perception 


\section{Einleitung}

Es ist unumstritten, dass Mathematik eine wesentliche Rolle in der kognitiven Entwicklung von Kindern spielt und mathematische Kompetenzen bereits in der frühen Kindheit angebahnt werden müssen (Schuler, Streit und Wittmann 2017). Um Mathematik im Alltag nutzen zu können, sind dabei günstige Ausprägungen affektiv-motivationaler Merkmale (z.B. hohe Freude, geringe Angst) förderlich (Jansen, Schmitz und van der Maas 2016; Schukajlow, Rakoczy und Pekrun 2017). Man kann davon ausgehen, dass der professionellen Kompetenz von frühpädagogischen Fachkräften eine besonders große Bedeutung in der affektivmotivationalen und mathematischen Kompetenzentwicklung von Kindern zukommt. Frühpädagogische Fachkräfte müssen somit während ihrer Ausbildung ihr eigenes mathematikspezifisches Wissen, sowohl bezüglich der Fachmathematik als auch der Mathematikdidaktik, spezifisch für die Bedürfnisse des Kita-Alltags aufbauen und erweitern (Gasteiger und Benz 2016). Die Identifikation mathematischer Potentiale einer Situation im Kita-Alltag kann als eine wesentliche Herausforderung für (angehende) frühpädagogische Fachkräfte angesehen werden (Dunekacke, Jenßen, Eilerts und Blömeke 2016; Wittmann, Böning, Levin und Schuler 2016).

Emotionen spielen für das professionelle Handeln von pädagogischen Fachkräften und somit auch im Rahmen deren Ausbildung eine bedeutende Rolle, z.B. in Praxisphasen oder in Prüfungssituationen (Porsch 2018). Studien zeigen, dass angehende frühpädagogische Fachkräfte sich in ihrer emotionalen Haltung zur Mathematik unterscheiden, manche berichten sogar von der Tendenz, mathematische Inhalte in ihrer beruflichen Laufbahn vermeiden zu wollen (Ginet, Itzkowich und Maloney 2018). Inwieweit eine derartige Mathematikangst auch praktische Bedeutung für den Kita-Alltag bei der professionellen Wahrnehmung mathematischer Potentiale in Situationen hat, wurde bislang jedoch nicht untersucht. Die Untersuchung des regressiven Zusammenhangs von Mathematikangst, professionellem Wissen im Bereich Mathematik und mathematikbezogener Situationswahrnehmung bei angehenden frühpädagogischen Fachkräften stellt somit ein aktuelles Forschungsdesiderat dar.

\section{Theorie}

\subsection{Emotionen in Lern- und Leistungsemotionen}

Im Kontext von Bildungsprozessen spielen Leistungsemotionen eine wesentliche Rolle (Pekrun und Linnenbrink-Garcia 2014; Pekrun, Muis, Frenzel und Goetz 2017). Pekrun (2006) führt in seiner Kontroll-Wert-Theorie aus, dass sich Emotionen neben biologischen Dispositionen (z.B. Gene, Temperament) im Wesentlichen auf Basis von grundlegenden Bewertungen einer spezifischen Situation ausformen. Lern- und Leistungssituationen, die sich beispielsweise in dem Niveau ihrer Anforderungen, in dem Grad der Autonomie, in den Zielstrukturen und Konsequenzen für das Individuum unterscheiden, werden hinsichtlich ihrer Kontrollierbarkeit (z.B. „Ich habe nicht das nötige mathematische Fachwissen, um eine mathematikhaltige Anforderung zu bewältigen.“) und ihrer Wertigkeit (z.B. „Mathematik ist ein bedeutsames Kulturgut") eingeschätzt. Ergebnis dieser Bewertungen stellt dann eine spezifische Leistungsemotion dar (z.B. Angst, die mathematische Situation nicht bewältigen zu können). Diese spezifische Leistungsemotion hat Effekte auf die kognitiven Ressourcen einer Person (z.B. Processing Efficiency Theory: gehemmte präfrontale kognitive Kapazität aufgrund intensiven negativen emotionalen Erlebens im limbischen System, z.B. Artemenko, Daroczy und Nuerk 2015; Young, Wu und Menon 2012), ihre Motivation und ihr weiteres Vorgehen (z.B. Lernstrategien, Selbstregulation). Neben allgemeinen kognitiven Fähigkeiten und Vorwissen zeigen die beschriebenen kurzfristigen Konsequenzen wiederum Effekte auf (langfristige) Lern- und Leistungsergebnisse. Pekrun (2006) beschreibt in seiner Theorie komplexe Rückkopplungen: So können sich eine gehemmte kognitive Kapazität oder ein negatives Ergebnis wiederum auf das emotionale Erleben auswirken. Mithilfe der KontrollWert-Theorie lassen sich die Entstehung von Emotionen und deren Wirkung beschreiben. 
Furchtbezogene Emotionen stellen dabei die mit am häufigsten untersuchten Leistungsemotionen dar.

\subsection{Mathematikangst}

Als eine solche furchtbezogene Emotion kann Mathematikangst gesehen werden. Mathematikangst wird definiert als ,feelings of tension and anxiety that interfere with the manipulation of mathematical problems in a wide variety of ordinary life and academic situations" (Richardson und Suinn 1972, S. 551). Bereits Ashcraft (2002) betonte, dass Mathematikangst, vergleichbar zu anderen Angstschemata, aus kognitiven und affektiven Komponenten besteht. Weiterhin muss davon ausgegangen werden, dass sich Mathematikangst nicht nur in Kognition und Emotion, sondern auch in Physiologie und Verhalten manifestiert (Bessant 1995).

Auf kognitiver Ebene zeigt sich Mathematikangst vor allem in dysfunktionalen Überzeugungen $\mathrm{zu}$ eigenem Versagen bei mathematikbezogenen Anforderungen (Hunt, Clark-Carter und Sheffield 2014; Ashcraft 2002). Als Primäremotion der Mathematikangst können Furcht und Scham angenommen werden, wobei die Bewertung dieser Primäremotionen in Sekundäremotionen wie Ärger, Hilflosigkeit und Frustration münden kann (Buxton 1981; Cherkas 1992). Physiologisch betrachtet führt Mathematikangst zu Anspannungsreaktionen, z.B. erhöhter Muskeltonizität oder vermehrtes Schwitzen (Hembree 1990). Darüber hinaus zeigt sich Mathematikangst auf Verhaltensebene vor allem in der Vermeidung mathematikhaltiger Anforderungen (Chang und Beilock, 2016), mathematiknaher Berufe (Chipman, Krantz und Silver 1992) oder der Wahl mathematikbezogener Kurse (Kelly und Tomhave 1985).

Mathematikangst kann sich in verschiedenen Situationen zeigen, die mathematikhaltige Anforderungen beinhalten (Cooke, Cavanaugh, Hurst und Sparrow 2011). Studien haben gezeigt, dass Mathematikangst bei hinreichender Manipulation situationsspezifische Schwankungen aufweisen kann (Bieg, Goetz, Wolter und Hall 2015; Goetz, Bieg, Lüdtke, Pekrun und Hall 2013). Diese Schwankungen können zudem geschlechtsspezifische Effekte aufweisen (ebd.). Generell wird für die Ausprägung von Mathematikangst ein geschlechtsspezifischer Effekt angenommen, wobei Frauen häufiger und ausgeprägter von Mathematikangst berichten (Hill et al. 2016; Sokolowski, Hawes und Lyons 2019; Porsch 2019).

Dass Mathematikangst bei angehenden als auch bei in der Praxis tätigen frühpädagogischen Fachkräften ein bedeutsames Phänomen darstellt, konnte bisher anhand mehrerer Studien für verschiedene Länder nachgewiesen werden (Aslan 2013; Gresham 2018; Jenßen, Dunekacke, Eid und Blömeke 2015; Thiel und Jenßen 2018). Die geschlechtsspezifischen Befunde zur Mathematikangst sind vor allem dahingehend bedeutsam, als dass die Mehrheit der frühpädagogischen Fachkräfte weiblich ist. Ähnlich wie angehende Grundschullehrkräfte weisen vermutlich auch angehende frühpädagogische Fachkräfte ein eher geringes Interesse an Mathematik auf (Porsch 2017) oder sie schätzen das fachmathematische Niveau der späteren Tätigkeit vorab vermutlich als zu gering ein (Porsch, Strietholt, Macharski und Bromme 2015), was bei beiden Berufsgruppen die höheren Ausprägungen von Mathematikangst erklären könnte (Ginet et al. 2018).

Porsch (2019) deutet in ihrem Review darüber hinaus auf die Bedeutung von Beliefs für Mathematikangst hin, die auch im Rahmen der oben beschriebenen Kontroll-Wert-Theorie eine Rolle spielen. Demnach weisen beispielsweise angehende Lehrkräfte höhere Ausprägungen von Mathematikangst auf, wenn sie das Fach als unbeliebt bewerten. Bewertungen zur Natur des Unterrichtens von Mathematik scheinen dem Review zu Folge dagegen in keinem Zusammenhang mit Mathematikangst zu stehen. Für frühpädagogische Fachkräfte in Deutschland konnte gezeigt werden, dass die Bewertung der Mathematik heterogen ausfällt, wobei die Mehrheit Mathematik als positiv bewertet (Thiel 2010; Benz 2012). Im Sinne der 
Kontroll-Wert-Theorie finden sich jedoch bisher keine empirischen Arbeiten zur Kontrollierbarkeitsdimension bezüglich mathematischer Anforderungen von (angehenden) frühpädagogischen Fachkräften.

Mathematikangst kann bei frühpädagogischen Fachkräften zu einer Vielzahl von Konsequenzen führen: Ausgehend von der Meta-Analyse von Ma (1999) lässt sich ein moderater negativer Zusammenhang zwischen ihrer Mathematikangst und ihrem mathematischen Wissen vermuten. Dazu gibt es mehrere Erklärungen: Zum einen führen mathematikbezogene Hinweisreize im Sinne der oben erwähnten Processing Efficiency Theory während der Bewältigung mathematischer Anforderungen $\mathrm{zu}$ einer Erhöhung der Reaktionszeiten und somit zu einem schlechten Abschneiden (Young et al. 2012; Ashcraft und Kirk 2001; Miller und Bichsel 2004). Nach dem Debilitating Anxiety Model (Chipman, Krantz, und Silver 1992; Hembree, 1990) vermeiden mathematikängstliche Personen mathematikhaltige Situationen und haben dadurch weniger Gelegenheiten, ihr mathematisches Wissen und Können zu erweitern. Der negative Effekt von Mathematikangst auf mathematisches Wissen wurde in einer Vielzahl von Studien untersucht (Carey, Hill, Devine und Szücs 2016) und kann auch für angehende frühpädagogische Fachkräfte angenommen werden (Jenßen al. 2015; Thiel und Jenßen 2018).

Dieses Defizit an mathematikspezifischem Wissen kann wiederum bedeuten, dass sie mathematische Kompetenzen der von ihnen betreuten Kindern nicht adäquat fördern können, mathematische Lerngelegenheiten nicht nutzen oder ihre eigene Mathematikangst auf die Kinder übertragen (Bates, Latham und Kim 2013; Beilock, Gunderson, Ramirez und Levine 2010; Gresham und Burleigh 2018; Mizala, Martínez und Martínez 2015). Ähnlich wie bei Primarstufenlehrkräfte, die nach dem Klassenlehrerprinzip unterrichten, werden auch frühpädagogische Lehrkräfte nicht fachspezifisch ausgebildet. Es ist daher davon auszugehen, dass Mathematikangst bei frühpädagogischen Fachkräften bedeutsam sein kann, da diese ihre Ausbildung nicht aus einem fachlichen Interesse an Mathematik wählen (Porsch, 2018). Im Unterschied zur Primarstufe stellt jedoch vor allem die potentielle Vermeidbarkeit mathematikhaltiger Situation im eher informellen und gering strukturierten Kita-Alltag eine mögliche schwerwiegende Konsequenz der Mathematikangst dar. Viele Bildungspläne für den Elementarbereich formulieren als Ziel, dass Kinder positive Erfahrungen mit Mathematik machen und Freude an Mathematik ausbilden sollen (vgl. Bayrisches Staatsministerium für Arbeit und Sozialordnung, Familie und Frauen 2016; Ministerium für Kultus, Jugend und Sport Baden-Württemberg 2011). Diese Freude kann nur jemand vermitteln, der selbst Freude an Mathematik hat (Karp 1991; Pólya 1977).

Die Frage, ob Mathematikangst auch eine praktische Bedeutsamkeit für angehende frühpädagogische Fachkräfte dahingehend besitzt, dass neben den Effekten auf mathematisches Wissen auch ein Zusammenhang zu handlungsnäheren Konstrukten wie der Situationswahrnehmung nachgewiesen werden kann, blieb bisher empirisch unbeantwortet.

\subsection{Mathematikspezifisches Wissen}

Mathematikspezifisches Wissen frühpädagogischer Fachkräfte kann als Element ihrer professionellen Kompetenz angesehen werden, genauer als professionelles Wissen im Bereich Mathematik. Gasteiger und Benz (2016) definieren das mathematikspezifische Wissen frühpädagogischer Fachkräfte als das Wissen, dass sich auf die konkrete mathematikspezifische Arbeit in der Kita bezieht. Ihrer Auffassung nach geht es um Kenntnisse im Bereich mathematischer Leitideen sowie Wissen über geeignete Materialien und ihre begründete Auswahl, Wissen über mathematische Entwicklungsprozesse von Kindern und deren Diagnose und Förderung. Deutlich wird dabei eine Differenzierung von mathematisch-fachlichem Wissen (MCK für mathematical content knowledge im internationalen Gebrauch) und mathematikdidaktischem Wissen (MPCK für mathematical pedagogical content knowledge im internationalen Gebrauch) (Depaepe, Verschaffel und Kelchtermans 2013). 
Allerdings gibt es bisher kein allgemein anerkanntes und wissenschaftlich fundiertes Curriculum, das dieses Wissen detailliert für die Frühpädagogik beschreibt (vgl. Blömeke et al. 2017). Um mathematikspezifisches Wissen frühpädagogischer Fachkräfte dennoch valide definieren und messen zu können, wurden deshalb von Jenßen, Dunekacke und Blömeke (2015) alle in Deutschland verwendeten Curricula für die Ausbildung frühpädagogischer Fachkräfte sowie die Bildungspläne der Bundesländer für den frühpädagogischen Bereich analysiert und als Basis für die Konstruktion der KomMa-Wissenstests verwendet, die auch in der vorliegenden Untersuchung zur Anwendung kamen (Blömeke et al. 2015b, 2017; Jenßen 2017). Alle Items wurden von Personen mit ausgewiesener Expertise in Bereichen früher mathematischer Bildung (Praxis, Ausbildung und Wissenschaft) auf ihre Relevanz für den frühpädagogischen Bereich überprüft (Jenßen, Dunekacke und Blömeke 2015).

\subsubsection{Mathematisches Fachwissen (MCK)}

MCK wird nach Ginsburg und Ertle (2008) benötigt, um das mathematische Wissen der Kinder einschätzen $\mathrm{zu}$ können. Hierfür ist ein breites Fachwissen über die verschiedenen mathematischen Inhalte hinweg nötig, welches aber auch innerhalb des jeweiligen Inhaltsbereiches in die Tiefe geht (Schoenfeld und Kilpatrick 2008). Deshalb umfasst der KomMa-MCK-Test von Blömeke et al. (2015b) die mathematischen Inhaltsbereiche (1) Zahlen, Mengen und Operationen, (2) Form, Raum und Veränderung, (3) Größen, Messen und Relationen sowie (4) Daten, Kombinatorik und Zufall. Die Anforderungen gehen weit über das hinaus, was von Kita-Kindern erwartet wird. Es handelt sich um Inhalte der Primarstufe, die als relevantes Hintergrundwissen für den frühpädagogischen Bereich angesehen werden. Abbildung 2 zeigt ein Beispiel. Kita-Kinder lösen eine solche Aufgabe enaktiv handelnd und unter Verwendung ikonischer Repräsentationen (Palmér und van Bommel 2018). Um sie in ihren Lernprozessen unterstützen zu können, wird von frühpädagogischen Fachkräften ein tieferes theoretisches Durchdringen der Situation erwartet.

Das mathematische Fachwissen von frühpädagogischen Fachkräften ist theoretischen Annahmen zufolge eine bedeutsame Facette ihrer professionellen Kompetenz im Bereich Mathematik (Anders 2012; Tsamir et al. 2014; Gasteiger und Benz 2016). Dennoch fokussierte die frühpädagogische Forschung in den vergangenen Jahren hauptsächlich das mathematikdidaktische Wissen von Fachkräften, auch wenn mittlerweile weitgehend Einigkeit darüber besteht, dass MCK das effektive Handeln der Fachkräfte in mathematikhaltigen Situationen maßgeblich mitbestimmt (Gasteiger und Benz 2016; Ginsburg und Ertle 2010; Klibanoff et al. 2006).

Forschungsarbeiten in Deutschland haben gezeigt, dass MCK einen hohen und praktisch bedeutsamen Zusammenhang mit dem mathematikdidaktischen Wissen bei angehenden frühpädagogischen Fachkräften aufweist und dass beide Wissensfacetten bedeutsam für die mathematikbezogene Situationswahrnehmung und Handlungsplanung im Kita-Alltag sind (Blömeke et al. 2015b; Dunekacke, Jenßen und Blömeke 2016). Es lässt sich aber auch feststellen, dass MCK bei angehenden frühpädagogischen Fachkräften eher auf einem niedrigen Niveau ausgeprägt ist (Blömeke et al. 2015b). In der Fachschulausbildung gibt es so gut wie keine Lerngelegenheiten, um für die Kita relevantes mathematisches Fachwissen zu erwerben (Blömeke et al. 2017). In der Regel beschränkt sich das MCK angehender frühpädagogischer Fachkräfte deshalb auf Wissen, das in der allgemeinbildenden Schule erworben wurde.

\subsubsection{Mathematikdidaktisches Fachwissen (MPCK)}

Neben MCK stellt das mathematikdidaktische Fachwissen eine weitere kognitive Facette professioneller Kompetenz von frühpädagogischen Fachkräften dar. MPCK beinhaltet nach Blömeke et al. (2015b) Wissen über geplante und situative Lerngelegenheiten im Bereich Mathematik und Wissen über die Entwicklung mathematischer Fähigkeiten bei Kindern. Es umfasst die Dimensionen „Entwicklung und Diagnose mathematischer Fähigkeiten bei Kindern im Alter von drei bis sechs Jahren“ und „Gestaltung situativer und geplanter Lernumgebungen 
im Bereich Mathematik in der Kita“ (Dunekacke et al. 2016). Im Gegensatz zu MCK wird MPCK konzeptionell eher als die „knowing-to-act“-Facette betrachtet (Depaepe et al. 2013), da es interaktionell geprägt und weniger faktischer Natur ist als MCK (Franke, Carpenter, Levi und Fennema 2001; Carpenter, Fennema, Peterson, Chiang und Loef 1989). Dies lässt sich eher mit offenen Aufgabenformaten erfassen als mit Multiple-Choice-Tests. Abbildung 3 zeigt ein Beispiel.

MPCK stellt die Grundlage dar, situative Bedingungen unter mathematischen Gesichtspunkten zu nutzen und mathematisches Denken bei Kindern anzuregen. Dunekacke et al. (2016) geben erste empirische Hinweise darauf, dass bei angehenden frühpädagogischen Fachkräften MCK als eine Vorbedingung für MPCK verstanden werden könnte (Depaepe et al. 2013). Dieses würde bedeuten, dass beide Wissensfacetten nicht lediglich korrelativ miteinander verbunden sind (z.B. durch Überlappungen auf Konstruktebene: Hill, Rowan und Ball 2005), sondern dass der Zusammenhang regressiver bzw. kausaler Natur sein könnte. MCK ist in dieser Vorstellung grundlegend für den Erwerb von MPCK (Ball 1988; Kahan, Cooper und Bethea 2003). Nach Lee, Meadows und Lee (2003) sind frühpädagogische Fachkräfte mit einem höheren MPCK eher dazu in der Lage, qualitativ hochwertigere Lerngelegenheiten im Kita-Alltag zu schaffen, als Fachkräfte mit niedrigerem MPCK.

\subsubsection{Zusammenhang mit der Mathematikangst}

Speziell zum Zusammenhang von Mathematikangst und mathematikspezifischem Wissen liegen bis heute nur zwei Studien für angehende frühpädagogische Fachkräfte vor. Jenßen et al. (2015) berichten für frühpädagogische Fachkräfte aus Deutschland einen signifikanten Zusammenhang zwischen Mathematikangst und MCK unter Berücksichtigung situationaler Schwankungen unter natürlichen Bedingungen. Im Zusammenhang mit MPCK liegen bisher keine Befunde vor. Thiel und Jenßen (2018) untersuchten den Effekt von Mathematikangst auf mathematikbezogenes Wissen bei angehenden frühpädagogischen Fachkräften in Norwegen, welches in der Abschlussprüfung abgefragt wurde, die sowohl fachmathematische als auch mathematikdidaktische Inhalte einschloss. Ähnlich wie in der Studie von Jenßen et al. (2015) konnte ein moderater negativer Effekt gefunden werden. Inwieweit aber Mathematikangst differenzielle Effekte auf MCK und MPCK zeigt, konnte in der Studie nicht untersucht werden.

\subsection{Mathematikbezogene Situationswahrnehmung}

Situationswahrnehmung für frühpädagogische Fachkräfte bedeutet das gezielte Erkennen möglicher relevanter Lernsituationen und des mathematischen Potenzials dieser Situation zur Umsetzung von spezifischen Förderstrategien (Morawiak, Schulz, Jungmann und Koch 2017). Dieses Erkennen erfordert fundiertes MCK und MPCK (Schuler, Wittmann, Levin und Bönig 2017; Dunekacke et al. 2016; McCray und Chen 2012). Situationswahrnehmung wird als eine von mehreren situationsspezifischen Fertigkeiten definiert und kann als integraler Bestandteil professioneller Kompetenz verstanden werden (Blömeke, Gustafsson und Shavelson 2015; Stahnke, Schüler und Rösken-Winter 2016, Santagat und Yeh, 2016). Situationsspezifischen Fertigkeiten kommt eine zentrale Bedeutung dabei zu, das vorwiegend deklarative MCK und auch MPCK in konkreten pädagogischen Handlungssituationen ,anwendbar“ zu machen, also Performanz zeigen zu können (Blömeke et al., 2015; Dunekacke et al., 2016; Kersting et al., 2010; Santagata und Yeh, 2016; Fröhlich-Gildhoff, Nentwig-Gesemann und Pietsch, 2011; Gasteiger und Benz, 2016). Dementsprechend stellt das professionelle Wissen für pädagogische Fachkräfte die Grundlage für eine effektive Situationswahrnehmung dar (Blömeke et al. 2014; Stahnke et al. 2016).

Aus der Wahrnehmungsforschung ist bekannt, dass Situationswahrnehmung im Wesentlichen in der Filterung der relevanten Informationen aus der Menge aller Informationen besteht (van Es und Sherin 2008; Goldstein 2002). Emotionen und Kognitionen (z.B. in Form von wissensbasierten Bewertungen) von pädagogischen Fachkräften sind an diesem Prozess maßgeblich beteiligt (Rosemann und Kerres 1985). Im Kontext früher (mathematischer) 
Bildung ist die Situationswahrnehmung dabei als funktional anzusehen, da sie die Grundlage für die Planung spezifischer, direkter didaktischer Handlungen bildet (Schweer 1992). Pädagogische Fachkräfte müssen eine Menge an Informationen wahrnehmen, um diese für ihr professionelles Handeln nutzen zu können (van Es und Sherin 2008). Gerade in der alltäglichen Kita-Praxis, in der konkrete Aktivitäten zur Förderung mathematischer Kompetenzen der Kinder nur in geringem Umfang planbar sind, kommt der professionellen Situationswahrnehmung dementsprechend erhebliche Bedeutung zu (Gasteiger und Benz 2016; Anders und Roßbach 2015; Fröhlich-Gildhoff et al. 2011).

Studien zeigen, dass sich die professionelle Situationswahrnehmung mit zunehmender beruflicher Erfahrung entwickelt (Star und Strickland 2008) und in Aus- und Weiterbildung trainierbar ist (Santagata und Yeh 2013; Santagata und Guarino 2011). Für angehende frühpädagogische Fachkräfte zeigt sich, dass die Ausprägung situationsspezifischer Fertigkeiten auf professionellem Wissen im Bereich Mathematik fußt und dass affektivmotivationale Orientierungen an dem Wahrnehmungsprozess beteiligt sind (Dunekacke et al. 2016; Lindmeier, Hepberger, Moser-Opitz und Heinze, 2016; McCray und Chen, 2012). MPCK zeigte einen bedeutsamen direkten positiven Einfluss auf die Situationswahrnehmung, während MCK einen bedeutsamen indirekten positiven Einfluss, vermittelt über MPCK, aufwies (Dunekacke et al., 2016). Blömeke und Jenßen (2017) konnten nachweisen, dass allgemeine kognitive Fähigkeiten erwartungsgemäß bei der Transformation von mathematikspezifischem Wissen in mathematikbezogene Situationswahrnehmung beteiligt sind.

Untersuchungsmethode der Wahl stellen für die Situationswahrnehmung videobasierte Testungen dar, da diese den situativen und zielgerichteten Charakter des Konstrukts valide abbilden können (Kersting et al. 2010; König et al. 2014; Kaiser et al. 2017). Solche videobasierten Verfahren finden mittlerweile auch in der Fortbildung frühpädagogischer Fachkräfte Anwendung (Bruns und Eichen 2018, Born-Rauchenecker, Drexl und Weber 2018). Für die Erfassung mathematikbezogener Situationswahrnehmung ist die Definition von objektivierbaren zugrundeliegenden Indikatoren wichtig. Diese können nach Thonhauser (2007) z.B. das Thema, das verwendete Material oder der soziale Kontext der Situation sein.

Im Zusammenhang mit Mathematikangst liegen - soweit bekannt - bisher keine Studien für pädagogische Fachkräfte vor. Basierend auf Einzelfallberichten ist jedoch davon auszugehen, dass es mathematikängstlichen frühpädagogischen Fachkräften schwerfällt, das mathematische Potenzial einer Situation einzuschätzen (Stoehr 2017). Ob dies allerdings nicht auch auf ein geringeres mathematisches Wissen zurückzuführen ist, blieb bisher ungeklärt. Studien mit einem Eye-Tracking-Ansatz zeigen ein spezielles Wahrnehmungsmuster, das bei Mathematikangst auftritt: mathematikängstliche Personen fixieren länger mathematische Inhalte und zeigen eine längere Verweildauer (Hunt, Clark-Carter und Sheffield 2015). Allerdings ist nicht davon auszugehen, dass eine tiefe Verarbeitung der Informationen erfolgt, sondern die Information im Sinne der Processing Efficiency Theory eher zu einer Reduktion kognitiver Kapazität bzw. kognitiver Vermeidung führt (Pizzie und Kraemer 2017; Young, Wu und Menon 2012). Insgesamt kann also festgehalten werden, dass bis auf wenige kognitionspsychologische Studien bisher keine mathematikdidaktischen Studien, speziell für frühpädagogische Fachkräfte, zu dem Zusammenhang von Situationswahrnehmung und Mathematikangst vorliegen.

\section{Studie}

\subsection{Forschungsfrage und Hypothesen}

Die vorliegende Studie hat zum Ziel die Bedeutung von Mathematikangst für die mathematikbezogene Situationswahrnehmung bei angehenden frühpädagogischen Fachkräften zu untersuchen. Darüber hinaus soll gegenüber anderen Studien zur Mathematikangst bei angehenden frühpädagogischen Fachkräften (Thiel und Jenßen 2018) die Frage geklärt werden, 
ob Mathematikangst differenzielle Effekte auf MCK und MPCK zeigt. Hierbei betrachten wir Mathematikangst in der von uns erfassten Form in Übereinstimmung mit der Forschungslage als (mehr oder weniger) konstante Persönlichkeitsdisposition (trait), die in der Regel während der Schulzeit erworben wird (Bekdemir 2010; Maloney und Beilock 2012).

Auf Basis von Konzeptionen der Emotionsforschung zur Mathematikangst (Buckley, Reid, Goos, Lipp und Thomson 2016; Ramirez, Shaw und Maloney 2018) und der Kompetenzforschung (z.B. Blömeke et al. 2015b; Gasteiger und Benz 2016) kann angenommen werden, dass Mathematikangst zyklische Wechselbeziehungen mit dem mathematikspezifischen Wissen einer angehenden frühpädagogischen Fachkraft aufweist (Carey, Hill, Devine und Szücs, 2016; Jansen et al. 2013). Wir beschränken uns hier auf die Untersuchung einer Richtung, nämlich angenommener direkter negativer Effekte der Mathematikangst auf MCK und MPCK, wie sie von der Processing Efficiency Theory (Artemenko, Daroczy und Nuerk 2015; Young, Wu und Menon 2012) und vom Debilitating Anxiety Model postuliert werden (Chipman, Krantz, und Silver 1992; Thiel und Jenßen 2018), um die spezifischen Effekte von Mathematikangst aufdecken zu können. Erst nachdem nachgewiesen wurde, dass Mathematikangst eine Rolle beim Erwerb professioneller Kompetenz spielt, macht es Sinn zyklische Beziehungen zu untersuchen, zum Beispiel wie durch entsprechende Ausbildungsinhalte Mathematikangst abgebaut werden könnte.

Anders und Roßbach (2015) konnten zeigen, dass Freude und Interesse an Mathematik einen direkten positiven Einfluss auf die Fähigkeit frühpädagogischer Fachkräfte haben, Mathematik in Spielsituationen zu erkennen. Es kann deshalb nicht ausgeschlossen werden, dass die Mathematikangst auch auf die Situationswahrnehmung einen direkten negativen Einfluss hat, der z.B. über das Debilitating Anxiety Model erklärt werden könnte (d.h. mathematikängstliche Personen meiden mathematikhaltige Situationen, haben deshalb weniger Erfahrungen mit solchen Situationen, weshalb es ihnen schwerer fällt, das mathematische Potenzial dieser Situationen einzuschätzen (vgl. Stoehr 2017)). Unser Strukturgleichungsmodell erlaubt es, direkte und indirekte Effekte der Mathematikangst vermittelt über die Wissensfacetten zu untersuchen und voneinander $\mathrm{zu}$ trennen. Eine Abschätzung des totalen Effekts der Mathematikangst wird daher möglich.

Erste empirische Befunde zu einzelnen Teilfragen stützen unsere Annahmen. Der komplexe Zusammenhang zwischen allen vier Variablen wurde jedoch bislang noch nicht empirisch untersucht. Entsprechend bisheriger Befunde und theoretischer Annahmen, gehen auch wir davon aus, dass MCK eine Vorbedingung von MPCK darstellt bzw. relevanter Prädiktor von MPCK ist (Dunekacke et al. 2016; Depaepe et al. 2013). Des Weiteren soll geprüft werden, ob beide Wissensfacetten einen direkten bzw. indirekten Effekt auf die Situationswahrnehmung zeigen. Das angenommene Modell wird in Abbildung 1 dargestellt.

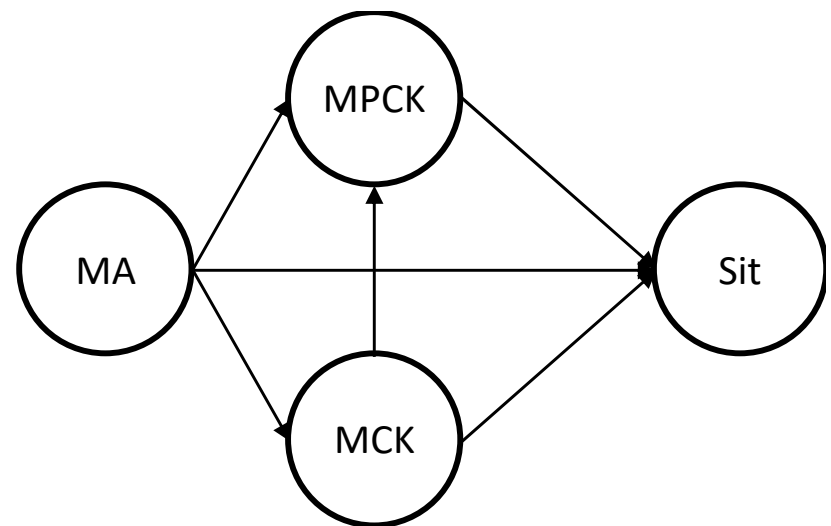

Abb. 1: Angenommenes Modell

Anm.: MA=Mathematikangst, $\mathrm{MCK}=$ mathematisches Fachwissen, MPCK=mathematikdidaktisches Wissen,

Sit=mathematikbezogene Situationswahrnehmung 


\subsection{Stichprobe}

In der vorliegenden Studie werden Daten aus dem Projekt $K o m M a^{1}$ (Jenßen et al. 2015) analysiert. Die Daten stammen von insgesamt $n=354$ angehenden frühpädagogischen Fachkräften aus Niedersachsen, Bremen und Berlin, die sich auf 16 Klassen von fünf Fachschulen verteilten. Das durchschnittliche Alter der Strichprobe betrug M = 22,9 Jahre (SD $=4,1$ Jahre). Der Großteil der Teilnehmenden gab an, weiblichen Geschlechts zu sein (83\%). Der weibliche Anteil entsprach somit dem Geschlechterverhältnis in der Population (BMFSFJ 2010, S. 13).

Die Teilnehmenden unterschieden sich in der Anzahl der bisher absolvierten Ausbildungsjahre: 41,5\% befanden sich zum Zeitpunkt der Erhebung in ihrem ersten Ausbildungsjahr, 33\% in ihrem zweiten und 25,5\% befanden sich am Ende ihrer Ausbildung. Diese drei Teilstichproben unterscheiden sich nicht wesentlich in ihrer Mathematikangst. Das stützt unsere Annahme, dass es sich um eine konstante Disposition handelt, die von der Fachschulausbildung in ihrer gegenwärtigen Form nicht beeinflusst wird.

Uns liegen keine Daten über fachmathematische und mathematikdidaktische Inhalte der betrachteten Fachschulen vor. Da es jedoch keine bedeutsamen Unterschiede im mathematischen Fachwissen der drei Teilstichproben gibt, liegt die Vermutung nahe, dass Mathematik in der Fachschulausbildung keine große Rolle spielt. Diese Annahme wird durch die wenigen empirischen Befunde zur Ausbildung frühpädagogischer Fachkräfte in Deutschland gestützt (Blömeke et al., 2017, KMK, 2017; Liebig, 2016; Kleeberger und Stadler, 2011). Im Unterschied dazu gibt es beim mathematikdidaktischen Wissen einen auf dem 5\%Niveau signifikanten Unterschied zwischen dem ersten und letzten Ausbildungsjahr. Der Unterschied beträgt etwa eine halbe Standardabweichung.

\subsection{Instrumente}

\subsubsection{Mathematics Anxiety Scale - Revised (MAS-R)}

Um Mathematikangst zu erfassen, wurde die revidierte Fassung der Mathematics Anxiety Scale von Bai et al. (2009) eingesetzt. Der Fragebogen besteht aus 14 Items, wobei acht Items negativ formulierte Aussagen darstellen (z.B. „Mathematik macht mich nervös“) und sechs Items positiv formuliert sind (z.B. „Ich finde Mathe interessant."). Den Autoren zufolge spiegeln die positiven Aussagen die kognitive Facette der Mathematikangst wider und die negativen Items die emotionale Facette. Die Teilnehmenden wurden gebeten die Aussagen auf einer 5-stufigen Likertskala von „trifft voll und ganz zu“ bis „trifft überhaupt zu“ einzuschätzen. Für den Gesamtscore wurden die Antworten aufsummiert, wobei die positiv formulierten Items invertiert wurden, sodass ein hoher Wert eine hohe Ausprägung an Mathematikangst widerspiegelt. Die von den Autoren angegebene Reliabilität (Cronbach's $\alpha=0,87$ ) kann als gut angesehen werden (zur Reliabilität der Skala in der vorliegenden Studie siehe Abschnitt 4.2). Es liegen Belege für valide Schlussfolgerungen des Fragebogens vor (Bai 2011). Der MAS-R erfasst Mathematikangst als trait. Analysen des Projekts haben gezeigt, dass der MAS-R auch unter natürlichen Bedingungen keine signifikanten Schwankungen im Sinne des state-Konzepts aufweist (Jenßen et al. 2015). Der Fragebogen wurde bereits auch bei anderen angehenden frühpädagogischen Fachkräften eingesetzt (Thiel und Jenßen 2018).

\subsubsection{KomMa-MCK-Test und KomMa-MPCK-Test}

Um das professionelle Wissen der angehenden frühpädagogischen Fachkräfte zu testen, wurden die umfassend validierten Leistungstests des Projekts KomMa eingesetzt (Blömeke et al. 2015b). Beide Tests bestehen sowohl aus Multiple-Choice-Aufgaben und Aufgaben, die ein offenes Antwortformat aufweisen (Open-Response-Items). Der KomMa-MCK-Test besteht aus

\footnotetext{
${ }^{1}$ KomMa war ein Kooperationsprojekt der Humboldt-Universität zu Berlin und der Alice-Salomon-Hochschule Berlin und wurde vom BMBF im Rahmen der Förderinitiative KoKoHs - Kompetenzmodellierung und Kompetenzerfassung im Hochschulsektor gefördert (FKZ: 01PK11002A).
} 
insgesamt 24 Items, wobei jeweils sechs Items einer mathematischen Domäne (Zahlen, Mengen und Operationen; Form, Raum und Veränderung; Größen, Messen und Relationen; Daten, Kombinatorik und Zufall) zugeordnet werden können. In Abbildung 2 ist ein Beispiel-Item des KomMa-MCK-Tests gegeben.

MPCK wurde mit einer Kurzskala des KomMa-MPCK-Tests erfasst, der im Rahmen von KomMa entwickelt wurde (Dunekacke et al. 2016). Die Kurzversion deckt alle relevanten Bereiche der ursprünglichen Langform ab und wurde aus testökonomischen Gründen gewählt. Die Kurzversion des KomMa-MPCK-Tests besteht aus 12 Items, die die Dimensionen „Entwicklung und Diagnose mathematischer Fähigkeiten bei Kindern im Alter von drei bis sechs Jahren" und "Gestaltung situativer und geplanter Lernumgebungen im Bereich Mathematik in der Kita" abdecken. Abbildung 3 zeigt ein Beispiel-Item des KomMa-MPCKTests, welches in einem offenen Format gestellt ist. Die Aufgaben beider Tests werden den dichotomen Kategorien richtig bzw. falsch zugeordnet. Die Interraterreliabilitäten für die Kodierung der Open-Response-Items liegen über beide Tests hinweg mindestens über einem $\kappa$-Wert von 0,88 .

Für beide Testverfahren liegt eine Vielzahl an Untersuchungen zu verschiedenen Validitätsaspekten vor. Sowohl der KomMa-MCK- als auch der KomMa-MPCK-Test wurde umfangreich anhand von IRT-basierten Analysen hinsichtlich Dimensionalität, Reliabilität und Itemeigenschaften untersucht. Eine umfassende Übersicht zu den IRT-Analysen findet sich bei Blömeke et al. (2015b) sowie bei Jenßen (2017) zu den Validierungen.

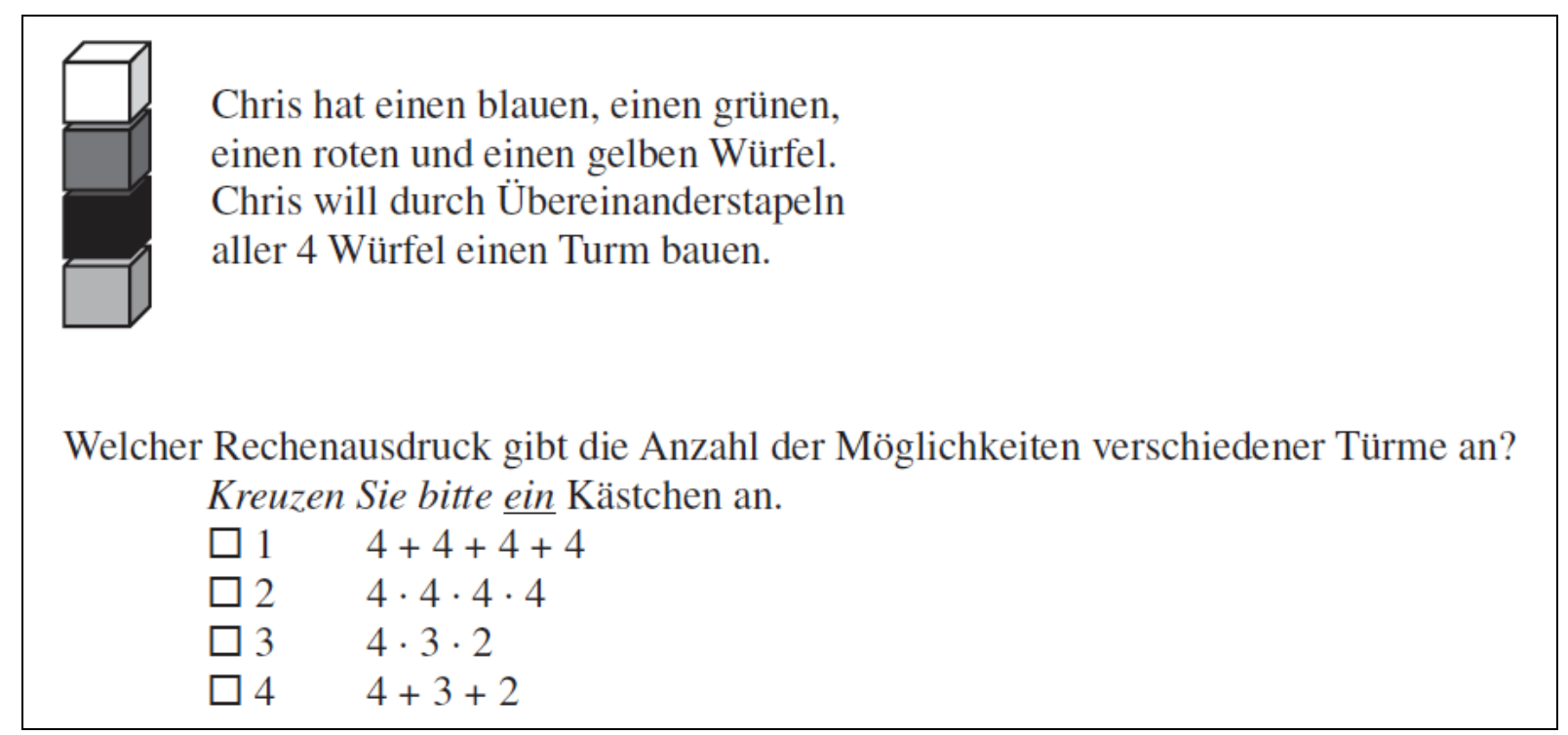

Abb. 2: Beispiel-Item des KomMa-MCK-Tests (richtige Lösung: 3) 


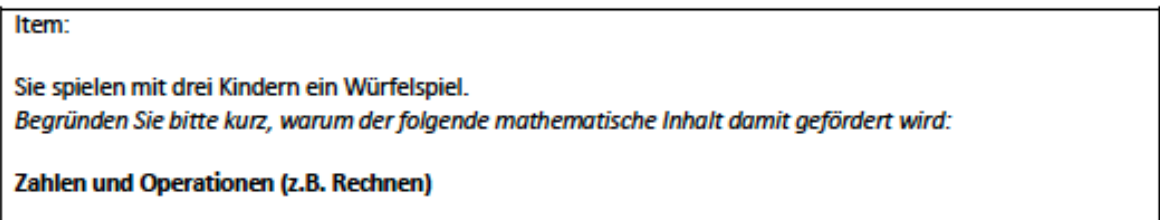

\begin{tabular}{l}
\hline Beispiele für richtige Antworten: \\
Mengen (Mengenvergleich, Mengenverständnis): \\
Antworten, die sich auf den konkreten Umgang mit Mengen beziehen, z.B. „Die Würfel haben Ziffern von \\
1-6, die Kinder lemen Mengenunterschiede der Punkte." \\
Simnltanes Erfassen: \\
Simnltanerfassung als Teil der Zahlbegiffsentwicklung. Kann auch umschrieben werden z. B. mit dem Verb \\
„erkennen“. "Sie müssen die Augenzahl auf dem Würfel erfassen “" \\
Eins-zu-eins-Zuordnung (durch Setzen der Spielfigur): \\
Alle Aussagen, bei denen es hauptsächlich darum geht, dass durch das Setzen der Spielfigur eine Zuordnung \\
zu der gewürfelten Zahl hergestellt werden muss, z.B. „Die Kinder erkennen eine Zahl auf dem Würfel und \\
setzen diese mit ihrer Figur auf dem Spielbrett um. "
\end{tabular}

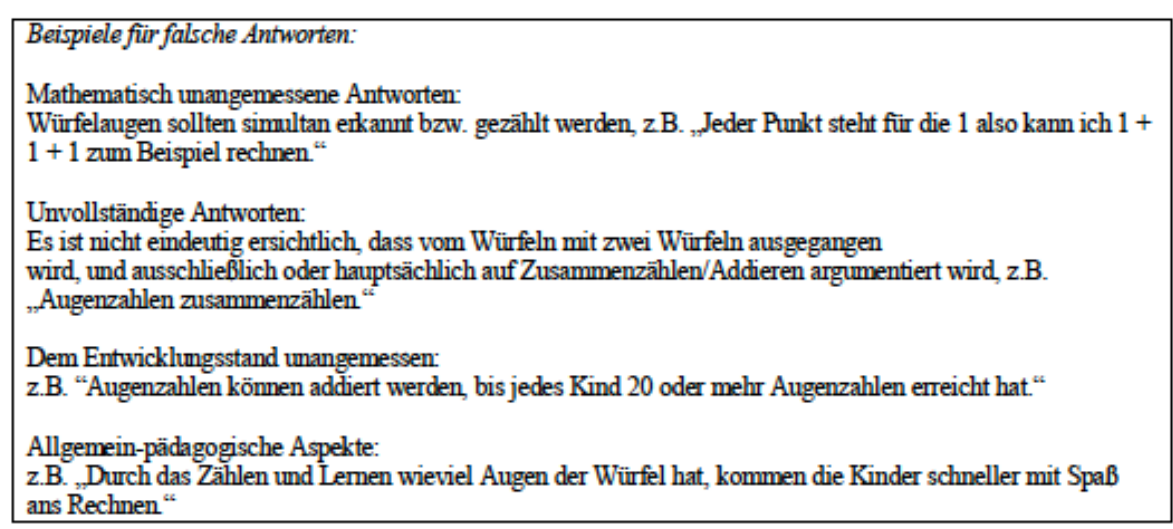

Abb. 3: Beispiel-Item des KomMa-MPCK-Tests

\subsubsection{Videobasierter Test zur Erfassung der Situationswahrnehmung}

Die mathematikbezogene Situationswahrnehmung wurde mittels eines videobasierten Tests erfasst, welcher von Dunekacke et al. (2016) entwickelt wurde und für den ebenfalls umfangreiche Validierungsanalysen vorliegen. Der Einsatz von Videovignetten ist in der Lehrerprofessionsforschung eine weitverbreitete Methodik (z.B. Kersting et al. 2010; Knievel, Lindmeier und Heinze 2015), kann bei den Teilnehmenden zu einer hohen Akzeptanz führen (Seidel und Prenzel 2007), erreicht eine hohe Situationsspezifität (Blomberg, Stürmer und Seidel 2011) und erlaubt unter bestimmten Voraussetzungen beine standardisierte Erfassung (König und Lebens 2012).

Der Test besteht aus drei Videos zu verschiedenen Domänen der frühen mathematischen Bildung (Zahldarstellungen, Messen, Bauen und Konstruieren). Die Videos, die im realen KitaKontext gedreht wurden, stellen verschiedene alltagstypische Situationen dar (z.B. Interaktion von frühpädagogischer Fachkraft und Kind, Kind im Freispiel). Zu jedem Video werden den Teilnehmenden vier identische Fragen gestellt (z.B. „Nennen Sie drei mathematikdidaktische Aspekte der Situation und belegen Sie sie mit Beispielen."). Die offenen Antworten werden anhand eines vorab systematisierten und validierten Code-Books den Antwortkategorien richtig bzw. falsch zugeordnet (für das konkrete Vorgehen: Dunekacke et al. 2016). Die Interraterreliabilität Yules Y liegt durchweg mindestens über einem Wert von 0,8 (ebd.).

\subsection{Datenerhebung}

Die Daten wurden im Rahmen des KomMa-Projektes (Jenßen et al. 2015) gewonnen, bei dem neben den hier betrachteten Konstrukten zu insgesamt drei Erhebungszeiten weitere Instrumente zum Einsatz kamen (z.B. Fragebogenverfahren zur Erfassung von allgemeinen und 
mathematikbezogenen Überzeugungen). Teile der in dieser Studie verwendeten Daten wurden daher bereits in anderen Arbeiten analysiert. Dunekacke et al. (2016) untersuchten beispielsweise mithilfe von selbst entwickelten und adaptierten Fragebögen die Effekte von Beliefs zum Wesen der Mathematik auf das mathematikbezogene Professionswissen und situationsspezifischen Fertigkeiten. In der vorliegenden Studie werden entsprechend der Fragestellung nun die Effekte der Mathematikangst untersucht. Die Skalen zur Erfassung der Beliefs zur Mathematik wurden in der vorliegenden Studie aus mehreren Gründen nicht einbezogen: Vor dem Hintergrund des begrenzten Stichprobenumfangs soll eine noch reliable Parameterschätzung der eingesetzten Konstrukte erlaubt werden. Zudem liefern die Beliefs, so wie sie im Projekt erhoben wurden, im Sinne der Kontroll-Wert-Theorie keinen informativen Gehalt zur Erklärung von Mathematikangst, vor allem hinsichtlich der Kontrollierbarkeit von Situationen (auch wenn sie darüber hinaus durchaus informativen Gehalt haben können). Zudem besteht das wesentliche Ziel der vorliegenden Studie darin, regressive Effekte allein von der Mathematikangst zu untersuchen. Die Teilnehmenden bearbeiteten zu einem ersten Messzeitpunkt den Fragebogen MAS-R und die beiden Leistungstests zur Erfassung des professionellen Wissens. Zu einem zweiten Messzeitpunkt wenige Tage später bearbeiteten die teilnehmenden angehenden frühpädagogischen Fachkräfte den videobasierten Test. Die Teilnehmenden wurden im Klassenverband schriftlich befragt und getestet. Zur Anreizteilnahme wurde pro Klasse ein Büchergutschein verlost. Die Datenerhebungen fanden durch geschulte Testleiterinnen und Testleiter statt, sodass die Durchführungsobjektivität gewährleistet werden konnte.

\subsection{Datenanalyse}

Um die in Abbildung 1 graphisch dargestellten Hypothesen testen zu können, wurde ein Strukturgleichungsmodell spezifiziert. Um das komplexe Modell angesichts der relativ kleinen Stichprobengröße zuverlässig testen zu können, wurde mit Item-Parceln gearbeitet (Little et al. 2002). Bei der Bildung der Parcel haben wir uns an der gängigen Praxis orientiert, indem wir darauf geachtet haben, dass statistische Kennwerte wie Mittelwerte der Items und Streuung über die Parcel gleichverteilt werden (ebd.). Zudem haben wir inhaltliche Begründungen bei dem Parceling beachtet: Um die Mathematikangst $\mathrm{zu}$ modellieren, wurde ein Parcel $\mathrm{zu}$ den ursprünglich positiv formulierten Items (kognitive Facette) und ein Parcel zu den negativ formulierten Items (emotionale Facette) gebildet. Das mathematische Fachwissen wurde durch vier Parcel repräsentiert, wobei jedes Parcel die Summe der Items bezüglich einer der vier mathematischen Domänen darstellt. Für das mathematikdidaktische Wissen wurden zwei Parcel entsprechend zweier Testhälften gebildet. Die inhaltlichen Dimensionen waren in beiden Testhälften gleichverteilt. Die Situationswahrnehmung wurde im Modell ebenfalls durch Parcel berücksichtigt: Die vier Antworten je Video wurden aufsummiert, sodass entsprechend der drei Videos drei Parcel das latente Konstrukt der Situationswahrnehmung abbildeten.

Die Daten wurden mithilfe der Software Mplus 8.1 (Muthén und Muthén 2017) analysiert. Die geclusterte Datenstruktur wurde anhand des Befehls TYPE=COMPLEX berücksichtigt. Die FIML-Prozedur erlaubte einen adäquaten Umgang mit fehlenden Werten, deren Anteil zwischen 17,5\% (für Mathematikangst) und 26\% (für MPCK) lag. Die Durchführung des Studienprotokolls lässt den Schluss zu, dass die Art des Datenausfalls mindestens missing at random ist. Trotz der erwartungsgemäß etwas höheren ausgeprägten Anteile der Missings kann demnach nach Enders (2010) davon ausgegangen werden, dass die FIML-Prozedur eine zuverlässige Schätzung der Parameter erlaubt. Variablen wie Wissen oder negative Emotionalität, die einen Ausfall der Daten nach missing at random erklären könnten, sind entsprechend der Empfehlung von Eid, Gollwitzer und Schmitt (2013) im Modell enthalten. 


\section{Ergebnisse}

\subsection{Deskriptive Ergebnisse}

Der mittlere Summenwert der MAS-R über alle Items lag bei $\mathrm{M}_{\mathrm{MA}}=43,78(\mathrm{SD}=10,71)$. Der mittlere Summenwert des MCK-Tests über alle Personen hinweg lag bei $\mathrm{M}_{\mathrm{MCK}}=11,46$ (SD = 4,23). Maximal wurden 23 von 24 möglichen Punkten erreicht. Der mittlere Summenwert des MPCK-Tests über alle Personen hinweg lag bei $\mathrm{M}_{\mathrm{MPCK}}=6,8(\mathrm{SD}=2,4)$. Maximal erreichten die angehenden frühpädagogischen Fachkräfte 11 von 12 möglichen Punkten. Die empirischen Mittelwerte liegen damit entsprechend der Testkonstruktion der Wissenstests im erwartbaren mittleren Bereich (Blömeke et al. 2015b). Der mittlere Summenwert für den videobasierten Test zur Situationswahrnehmung lag bei $\mathrm{M}_{\mathrm{Sit}}=6,34(\mathrm{SD}=2,00)$ bei einem maximal erreichbaren Wert von 12 Punkten. Tabelle 1 gibt einen Überblick über die deskriptiven Ergebnisse zu den manifesten Indikatoren.

Tab. 1: Mittelwerte, Standardabweichungen, Minima und Maxima, Faktorladungen und erklärte Varianz $\left(\mathrm{R}^{2}\right)$ der Indikatoren bzw. Parcel

\begin{tabular}{|c|c|c|c|c|c|c|c|}
\hline Indikator & $\mathrm{M}$ & SD & Min & Max & $\lambda$ & $\mathrm{R}^{2}$ & Faktor \\
\hline $\mathrm{ZMO}$ & 3,29 & 1,42 & 0 & 6 & 0,73 & 0,54 & \multirow[t]{4}{*}{ MCK } \\
\hline FRV & 2,74 & 1,50 & 0 & 6 & 0,74 & 0,55 & \\
\hline GMR & 2,65 & 1,19 & 0 & 6 & 0,66 & 0,44 & \\
\hline DKZ & 2,77 & 1,33 & 0 & 6 & 0,61 & 0,37 & \\
\hline $\mathrm{MPCK}_{1}$ & 3,58 & 1,53 & 0 & 6 & 0,70 & 0,49 & \multirow[t]{2}{*}{ MPCK } \\
\hline $\mathrm{MPCK}_{2}$ & 3,70 & 1,41 & 0 & 6 & 0,72 & 0,51 & \\
\hline MAp & 19,86 & 5,32 & 7 & 30 & 0,84 & 0,71 & \multirow{2}{*}{$\begin{array}{l}\text { Mathe- } \\
\text { Angst }\end{array}$} \\
\hline MAn & 23,91 & 6,71 & 8 & 40 & 0,69 & 0,48 & \\
\hline $\mathrm{SitV}_{1}$ & 2,24 & 0,84 & 0 & 4 & 0,52 & 0,27 & \multirow{3}{*}{$\begin{array}{c}\text { Situations- } \\
\text { wahrnehmung }\end{array}$} \\
\hline $\mathrm{SitV}_{2}$ & 2,09 & 1,02 & 0 & 4 & 0,50 & 0,25 & \\
\hline $\mathrm{SitV}_{3}$ & 2,02 & 0,93 & 0 & 4 & 0,58 & 0,33 & \\
\hline
\end{tabular}

Anm.: ZMO - Zahlen, Mengen und Operationen, FRV - Form, Raum und Veränderung, GMR - Größen, Messen und Relationen, DKZ - Daten, Kombinatorik und Zufall, MPCK - Testhälfte i des KomMa-MPCK-Tests, MAp - positiv formulierte Mathematikangst, MAn - negativ formulierte Mathematikangst, $\mathrm{Sit}_{\mathrm{i}}$ - Video i des Tests zur Erfassung der Situationswahrnehmung, M - Mittelwert, SD - Standardabweichung, Min - Minimum, Max - Maximum, $\lambda$ - standardisierte Ladung, $\mathrm{R}^{2}$ - aufgeklärte Varianz des Indikators durch den entsprechenden Faktor

Tabelle 2 zeigt die Varianzen und bivariaten Zusammenhänge der latenten Variablen an. Mathematikangst hängt erwartungsgemäß und signifikant $(p<0,001)$ mit allen drei Kompetenzfacetten zusammen, am deutlichsten mit MCK. Die Kompetenzfacetten weisen untereinander latente Korrelationen mittlerer Stärke auf. Alle angegebenen Korrelationen sind signifikant $(p<0,001)$.

Tab. 2: Varianzen (diagonal) und Korrelationen der latenten Variablen

\begin{tabular}{|c|c|c|c|c|}
\hline & MA & MCK & MPCK & Sit \\
\hline MA & 19,96 & & & \\
\hline MCK & $-0,45$ & 1,08 & & \\
\hline MPCK & $-0,17$ & 0,675 & 1,14 & \\
\hline Sit & $-0,23$ & 0,50 & 0,67 & 0,19 \\
\hline
\end{tabular}

Anm.: MA=Mathematikangst, MCK=mathematisches Fachwissen, MPCK=mathematikdidaktisches Wissen, Sit=mathematikbezogene Situationswahrnehmung

\subsection{Reliabilität der Skalen und Passung des Strukturgleichungsmodells}

Zur Schätzung der Reliabilität wurde für jede Skala McDonald's $\omega$ berechnet (Eid et al. 2013). Für die Skala zur Erfassung der Mathematikangst beträgt die Reliabilität $\omega_{\mathrm{MA}}=0,73$ Für die 
beiden KomMa-Tests liegen ebenfalls akzeptable Reliabilitätswerte vor mit $\omega_{\mathrm{MCK}}=0,78$ und $\omega_{\mathrm{MPCK}}=0,67$. Die Reliabilität des Videotests zur Erfassung des mathematikbezogenen Wissens fiel mit $\omega_{\mathrm{Sit}}=0,54 \mathrm{zwar}$ niedrig, aber erwartbar für videobasierte Kompetenztests aus. Alle Ladungen der Indikatoren im Strukturgleichungsmodell waren substanziell und signifikant $(p<0,001)$. Der Model Fit kann mit $\chi^{2}(38)=47,92, p=0,13, \operatorname{RMSEA}=0,02 \quad[0,00 ; 0,05]$, $\mathrm{SRMR}=0,03$ und $\mathrm{CFI}=0,99$ als sehr gut bewertet werden. Das empirische Strukturgleichungsmodell mit den Varianzen der latenten Variablen (in Klammern) und Regressionsgewichten ist in Abbildung 4 dargestellt. Die standardisierten Faktorladungen der jeweiligen Indikatoren sind in Tabelle 1 angegeben.

\subsection{Effekte von Mathematikangst}

Mathematikangst zeigte einen signifikant moderaten bis starken negativen Effekt auf MCK $\left(\beta_{M A, M C K}=-0,45, p<0,001\right)$. Um zu veranschaulichen, was dies bedeutet, stellen wir Personen, die hochmathematikängstlich sind (d.h. Personen, deren Mathematikangst mindestens eine Standardabweichung über dem gemessenen Mittelwert liegt) niedrigmathematikängstlichen Personen (d.h. Personen, deren Mathematikangst mindestens eine Standardabweichung unter dem gemessenen Mittelwert liegt) gegenüber (vgl. Ramirez et al. 2018). Der mittlere Summenwert des MCK-Tests von niedrigmathematikängstlichen Personen ist etwa eine Standardabweichung höher als der mittlere Summenwert des MCK-Tests von hochmathematikängstlichen Personen $(14,09$ vs. 10,$11 ; t=5,378 ; d f=101,888 ; p<0,001)$.

Direkte Effekte der Mathematikangst auf MPCK und auf die mathematikbezogene Situationswahrnehmung konnten nicht nachgewiesen werden. Auf die Situationswahrnehmung vermittelt über MPCK und MCK zeigte sich allerdings ein signifikanter indirekter negativer Effekt der Mathematikangst von $\beta_{\text {ind } 1}=-0,22(p<0,05)$. Der mittlere Summenwert für den videobasierten Test zur Situationswahrnehmung lag für Niedrigmathematikängstliche etwa eine halbe Standardabweichung höher als der Wert für Hochmathematikängstliche (7,02 vs. $6,02 ; t=2,525 ; d f=86 ; p<0,05)$.

Auf MPCK vermittelt über MCK konnte ein signifikanter negativer indirekter Effekt der Mathematikangst von moderater Größe gefunden werden $\left(\beta_{\text {ind } 2}=-0,34 ; p<0,001\right)$. Der Unterschied zwischen niedrig- und hochmathematikängstlichen Personen beträgt auch hier etwa eine halbe Standardabweichung (7,77 vs. 6,50; $t=2,554 ; d f=80 ; p<0,05)$.

\subsection{Effekte des mathematikspezifischen Wissens}

Entsprechend unserer Annahme haben wir MCK als Prädiktor von MPCK im Strukturgleichungsmodell spezifiziert. Der Regressionseffekt kann mit $\beta_{M C K, M P C K}=0,75$ $(p<0,001)$ als stark positiv bewertet werden. MPCK zeigte einen starken direkten positiven Effekt auf die mathematikbezogene Situationswahrnehmung $\left(\beta_{M P C K, S i t}=0,64, p<0,001\right)$. Es konnte kein direkter signifikanter Effekt von MCK auf die Situationswahrnehmung festgestellt werden. Allerdings zeigt sich ein indirekter, über MPCK vermittelter, positiver Effekt von $\beta_{\text {indMCK }}=0,48(p<0,01)$, der von moderater Stärke war. 


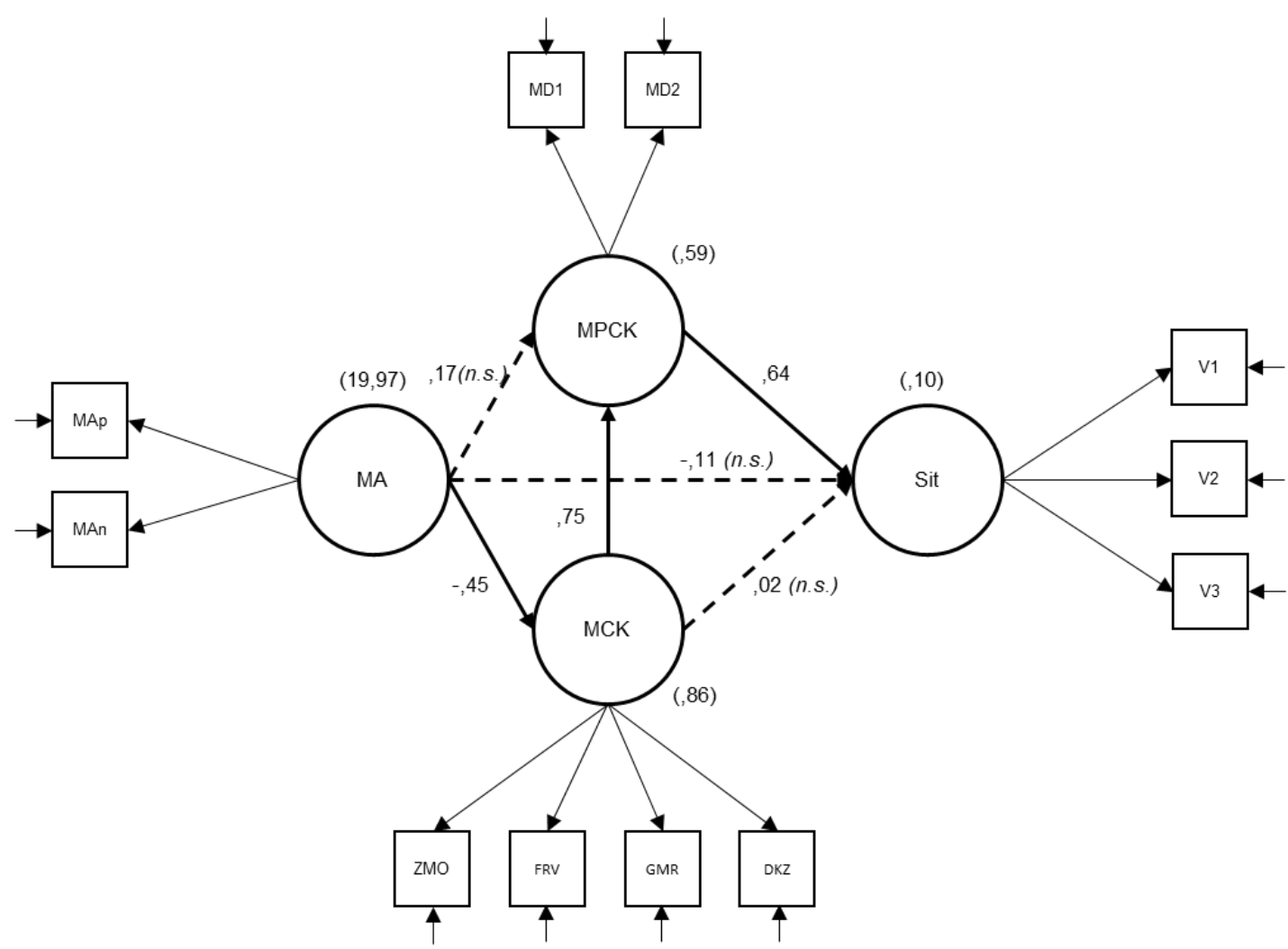

Abb. 4: Empirisch ermitteltes Strukturgleichungsmodell

Anm.: MA=Mathematikangst, MAp=positiv formulierte Items Mathematikangst, MAn=negativ formulierte Mathematikangst, MCK=mathematisches Fachwissen, ZMO=Parcel zu dem Inhalt Zahlen, Mengen und Operationen, FRV=Parcel zu dem Inhalt Form, Raum und Veränderung, GMR=Parcel zu dem Inhalt Größen, Messen und Relationen, DKZ=Parcel zu dem Inhalt Daten, Kombinatorik und Zufall, MPCK=mathematikdidaktisches Wissen, MD1=Parcel zur ersten Testhälfte des MPCK-Tests, MD2=Parcel zur zweiten Testhälfte des MPCK-Tests, Sit=mathematikbezogene Situationswahrnehmung, Vi=Parcel des i-ten Videos des videobasierten Tests zur Erfassung mathematikbezogener Situationswahrnehmung, Es werden durchweg standardisierte Koeffizienten berichtet.

\section{Diskussion}

Die vorliegende Studie betont die Bedeutsamkeit affektiv-motivationaler Dispositionen für die professionelle Kompetenz von angehenden frühpädagogischen Fachkräften. Die Ergebnisse deuten dabei darauf hin, dass Mathematikangst ausschließlich über die Vermittlung der Wissensfacetten Effekte auf die Situationswahrnehmung hat. Vor dem Hintergrund anderer Studien (Dunekacke et al. 2016), ist davon auszugehen, dass Mathematikangst den Erwerb von mathematischem Fachwissen behindert, welches wiederum wichtig für den Erwerb von mathematikdidaktischem Wissen ist, welches schließlich Effekte auf die professionelle Wahrnehmung mathematischer Potenziale einer Situation in der Kita-Praxis hat. Über diese angenommene Kette konnte ein signifikanter indirekter Effekt von Mathematikangst auf die Situationswahrnehmung festgestellt werden. Die Angst vor Mathematik scheint also nicht nur für die Ausprägung des mathematikbezogenen Wissens sowohl direkt (MCK) als auch indirekt (MPCK) bedeutsam zu sein, sondern auch nachweisbar dafür, wie frühpädagogische Fachkräfte mathematikbezogene Situationen wahrnehmen. Die indirekten bzw. direkten Effekte von MCK und MPCK auf die Situationswahrnehmung, die auch in der Arbeit von Dunekacke et al. (2016) berichtet werden, stellen sich auch unter Kontrolle von Mathematikangst in der vorliegenden Studie als stabil dar. Die Vorhersagekraft von MCK auf MPCK ist durch die Aufnahme von Mathematikangst sogar stärker $\left(\beta_{M C K, M P C K}=0,75\right)$ als bei der Modellierung mit Beliefs zum Wesen der Mathematik ( $\beta_{M C K, M P C K}=0,55$; vgl. Dunekacke et al. 2016).

Entgegen unserer Hypothese konnten wir keine direkten Effekte der Mathematikangst auf MPCK und auf die Situationswahrnehmung feststellen. Dies könnte daran liegen, dass MPCK 
und die mathematikbezogene Situationswahrnehmung nicht direkt fachmathematische Aufgaben in den Vordergrund stellen, sondern sich deutlicher als MCK auf den frühpädagogischen Bereich beziehen, der sich strukturell und inhaltlich von der Schule unterscheidet. Mathematikhaltige Situationen im frühpädagogischen Bereich werden unter Umständen von mathematikängstlichen Personen nicht in gleicher Weise als beängstigend wahrgenommen, wie dies bei schulmathematikhaltigen Situationen der Fall ist. Dies könnte unter anderem daran liegen, dass es den frühpädagogischen Fachkräften aufgrund geringeren mathematischen Fachwissens schwerer fällt, solche Situationen überhaupt als mathematikhaltig zu erkennen. Diesen Schluss legt der indirekte Einfluss der Mathematikangst über das Fachwissen auf die Situationswahrnehmung nahe.

Der Befund, dass die Mathematikangst indirekten Einfluss auf MPCK und die Situationswahrnehmung hat, ist bedeutsam und hat praktische Konsequenzen. Auch wenn Mathematikangst die angehenden frühpädagogischen Fachkräfte nicht direkt daran hindert, für die Arbeit mit Mathematik nötiges Handlungswissen zu erwerben, so hat sie doch einen Effekt. Der Befund, dass angehende frühpädagogische Fachkräfte die Mathematikhaltigkeit typischer Kita-Situationen umso besser wahrnehmen, je mehr mathematisches Fachwissen sie haben, legt die Vermutung nahe, dass sich die Behandlung fachmathematischer Inhalte in der Ausbildung positiv auf diese Fähigkeit auswirken könnte. Unseren Befunden zufolge, spielt dabei die Mathematikangst eine Rolle. Der nachgewiesene negative Effekt der Mathematikangst auf MCK stützt Theorien, die die negativen Auswirkungen von Mathematikangst auf den Erwerb mathematischen Wissens erklären (insbesondere die Processing Efficiency Theory, z.B. nach Artemenko, Daroczy und Nuerk 2015 und Young, Wu und Menon 2012, und das Debilitating Anxiety Model, z.B. nach Chipman, Krantz und Silver 1992).

Ausgehend von der Kontroll-Wert-Theorie und empirischen Befunden zu Beliefs lässt sich ableiten, dass angehende frühpädagogische Fachkräfte die eigene Mathematikangst reduzieren können, umso mehr sie der Überzeugung sind, mathematikhaltige Situationen (fachlich und didaktisch) bewältigen zu können. Diese Überzeugung könnte dadurch gestärkt werden, indem sie ihr Wissen erweitern und vor allem beispielsweise erkennen, welches mathematische Fachwissen sie speziell für die Kita-Praxis benötigen. Faktisch herrscht hierzu aber keine Einigkeit in der Forschung und Praxis (Jenßen 2017). Die Frage, welches mathematische Fachwissen frühpädagogische Fachkräfte benötigen, ist auch im Ansatz nicht hinreichend geklärt. Auch hier wären ausgearbeitete Konzepte und empirische Studien in der Zukunft unabdingbar.

Das Ergebnis der Untersuchung legt die Vermutung nahe, dass sich eine Reduktion von Mathematikangst positiv auf den Wissenserwerb und damit indirekt auch auf die Situationswahrnehmung auswirken würde. Thiel (2010) und Thiel und Jenßen (2018) vermuten aufgrund ihrer Befunde, dass sich positive Erfahrungen mit mathematikhaltigen Situationen in der Kita positiv auf die Haltung zur Mathematik auswirken könnten. Dies kann letztendlich nur mit Interventionsstudien geprüft werden, die auch kausale Aussagen ermöglichen. Unsere Ergebnisse sollten als Hinweise zu Effekten verstanden werden, die für die Planung und Durchführung solcher Studien beitragen.

Inhalten und Struktur der Ausbildung von frühpädagogischen Fachkräften kommen auch vor dem Hintergrund der vorliegenden Studie eine zentrale Bedeutung zu (Blömeke et al. 2017). Eine Reduktion von Mathematikangst würde sicherlich davon profitieren, wenn Mathematik expliziter Bestandteil der Ausbildung ist und systematische Lerngelegenheiten geschaffen werden. Aus der Diskussion um (fachfremd unterrichtende) Primarstufenlehrkräfte ist davon auszugehen, dass ein klares fachmathematisches Interesse mit einer geringen Mathematikangst einhergeht (Porsch 2018). Mathematikangst kann jedoch wohlmöglich nicht nur über mathematikspezifische Lerngelegenheiten reduziert werden, sondern bedarf in einem ersten Schritt der emotional-kognitiven Reflexion. Diese kann und sollte ebenso Teil der Ausbildung von frühpädagogischen Fachkräften sein. 
Die Ergebnisse der vorliegenden Studie sind jedoch vor dem Hintergrund einiger Limitationen zu interpretieren. So ist die Repräsentativität dahingehend eingeschränkt, da es sich um eine Gelegenheitsstichprobe handelt und die Stichprobe nicht angehende frühpädagogische Fachkräfte aus allen Bundesländern umfasst. Die Kurzversion des KomMa-MPCK-Tests deckt zwar alle relevanten Bereiche der ursprünglichen Langform ab. Wegen der geringeren Anzahl von Items kann eine Konstruktunterrepräsentation jedoch nicht vollkommen ausgeschlossen werden (Jenßen 2017). Neben der eingeschränkten Repräsentativität möchten wir nochmals darauf verweisen, dass die Annahmen in unserem Modell regressiver Natur sind, um Effekte aufzudecken, die explizit auf Mathematikangst zurückzuführen sind. Dennoch erlauben unsere Ergebnisse selbstverständlich keine kausalen Interpretationen, da unserer Studie ein querschnittliches Design zugrunde liegt. Um kausale Aussagen zu ermöglichen, wären, wie bereits erwähnt, zukünftige Interventionsstudien, möglichst in einem längsschnittlichen Design, nötig.

Einschränkend sind in unserer Studie auch die niedrigen Reliabilitäten bzw. Varianzaufklärungen der Indikatoren, insbesondere für die mathematikbezogene Situationswahrnehmung, zu nennen. Dies kann zu einer Varianzeinschränkung bei der latenten Variablen führen, welche wiederum anzeigt, dass Unterschiede zwischen Personen mithilfe des Instruments unter Umständen nicht deutlich gemacht werden (Lord und Novick 1968). Die niedrigen Reliabilitäten der Indikatoren spiegeln dabei auch das Reliabilititäts-ValiditätsDilemma wider, welches insbesondere bei Kompetenzmessungen auftreten kann (Rost 2008).

Mathematikangst wurde in der vorliegenden Untersuchung als eindimensionales Konstrukt konzeptualisiert und gemessen und nicht als kategoriale Diagnose verstanden. Sie beschreibt hier beispielsweise nicht eine pathologische absolute Vermeidung von mathematikspezifischen Situationen (z.B. Faust 1992), sondern eine eingeschränkte Hinwendung zu mathematischen Inhalten (Ashcraft 2002). Bei der Erforschung von Mathematikangst ist es nicht selten, dass Personen, die hochmathematikängstlich sind, niedrigmathematikängstlichen Personen gegenübergestellt werden (Ramirez et al. 2018). In der vorliegenden Studie wird dieser Vergleich nur zur Veranschaulichung der Effektstärken herangezogen. Im Pfadmodell wurden jeweils alle Probanden berücksichtigt.

Zudem wurde Mathematikangst nicht speziell auf frühe mathematische Bildung bezogen erfasst. Dies ist rein konzeptionell keine Einschränkung, da sich Mathematikangst immer auf alle mathematischen Bereiche bezieht (u.a. Ramirez et al. 2018). Dies macht die vorliegende Studie deutlich: Eine allgemeine Mathematikangst wirkt sich auf die mathematikbezogenen Kompetenzfacetten frühpädagogischer Fachkräfte aus, die direkt handlungsrelevant sind. Zusätzlich wäre es interessant zu prüfen, ob sich die Effekte verändern, wenn Instrumente zur Erfassung von Mathematikangst zum Einsatz kommen, die sich speziell auf frühe mathematische Bildung beziehen. Aktuell liegen derartige standardisierte und empirisch erprobte Instrumente unseres Wissens jedoch nicht vor.

Auch unter Berücksichtigung der aufgeführten Beschränkungen ist angesichts der sorgfältigen Verankerung unserer Studie in etablierten theoretischen Modellen davon auszugehen, dass die Ergebnisse relativ robust sind und Mathematikangst damit eine praktische Bedeutung für die professionelle Kompetenz angehender frühpädagogischer Fachkräfte hat - insbesondere hinsichtlich deren Wissens und situationsspezifischer Fertigkeiten. Die Befunde unserer Studie geben damit, respektive der gegebenen Limitationen, weitere Hinweise, dass das Thema frühe mathematische Bildung in der Ausbildung von frühpädagogischen Fachkräften einer expliziten (Gasteiger und Benz 2016) und fachlich fundierten Behandlung bedarf (Mischo 2016; Blömeke et al. 2017). Hierbei deuten unsere Befunde darauf hin, dass es wichtig ist, neben Aspekten der frühen mathematischen Bildung (MPCK und Situationswahrnehmung) auch das mathematische Fachwissen und damit verbundene Ängste methodisch-didaktisch adäquat anzugehen, um den Fachkräften so eine Weiterentwicklung ihres Wissens und ihrer Fähigkeiten zu ermöglichen. 
Eine Reihe von Fragen bleibt offen: Inwieweit hat Mathematikangst, ggf. vermittelt über die hier untersuchten Konstrukte, auch Effekte auf das tatsächliche professionelle Handeln angehender Fachkräfte im Kita-Alltag? Wie bedeutsam ist Mathematikangst bei frühpädagogischen Fachkräften, die bereits in der Praxis tätig sind? Welchen Effekt hat Mathematikangst von frühpädagogischen Fachkräften auf die Entwicklung mathematischer Kompetenzen bei Kindern? Zukünftige Forschung sollte auf diese Fragen fokussieren.

\section{Literatur}

Anders, Y. (2012). Modelle professioneller Kompetenzen für frühpädagogische Fachkräfte. Aktueller Stand und ihr Bezug zur Professionalisierung. Expertise zum Gutachten „Professionalisierung in der Frühpädagogik“. Aktionsrat Bildung.

Anders, Y. \& Rossbach, H.-G. (2015). Preschool Teachers' Sensitivity to Mathematics in Children's Play. The Influence of Math-Related School Experiences, Emotional Attitudes, and Pedagogical Beliefs. Journal of Research in Childhood Education, 29(3), 305-322.

Artemenko, C., Daroczy, G., \& Nuerk, H.-C. (2015). Neural correlates of math anxiety - an overview and implications. Frontiers in Psychology, 6(September). https://doi.org/10.3389/fpsyg.2015.01333

Ashcraft, M. H. \& Kirk, E. P. (2001). The relationships among working memory, math anxiety, and performance. Journal of Experimental Psychology: General, 130(2), 224-237.

Ashcraft, M.H. (2002). Math Anxiety: Personal, Educational, and Cognitive Consequences. Current Directions in Psychological Science, 11(5), 181-185.

Bai, H. (2011). Cross-Validating a Bidimensional Mathematics Anxiety Scale. Assessment, $18(1), 115-122$.

Bai, H., Wang, L.S., Pan, W., \& Frey, M. (2009). Measuring Mathematics Anxiety: Psychometric Analysis of a Bidimensional Affective Scale. Journal of Instructional Psychology, 36(3), 185-193.

Ball, D. (1988). Research on teaching mathematics: making subject matter knowledge part of the equation. East Lansing: National Center for Research on Teacher Education.

Barnhart, T. \& van Es, E. (2015). Studying teacher noticing: Examining the relationship among pre-service science teachers' ability to attend, analyze and respond to student thinking. Teaching and Teacher Education, 45, 83-93.

Bates, A. B., Latham, N. I., \& Kim, J. (2013). Do I Have to Teach Math? Early Childhood PreService Teachers' Fears of Teaching Mathematics. IUMPST: The Journal, 5(August).

Bayerisches Staatsministerium für Arbeit und Sozialordnung, Familie und Frauen, \& Staatsinstitut für Frühpädagogik (2016). Der Bayerische Bildungs- und Erziehungsplan für Kinder in Tageseinrichtungen bis zur Einschulung (7. Aufl.). Berlin: Cornelsen.

Bekdemir, M. (2010). The pre-service teachers' mathematics anxiety related to depth of negative experiences in mathematics classroom while they were students. Educational Studies in Mathematics, 75(3), 311-328.

Beilock, S. L., Gunderson, E. A., Ramirez, G., \& Levine, S. C. (2010). Female teachers' math anxiety affects girls' math achievement. Proceedings of the National Academy of Sciences, 107(5), 1860-1863. https://doi.org/10.1073/pnas.0910967107

Benz, C. (2012). "Maths is not dangerous" - Attitudes of people working in German kindergarten about mathematics in kindergarten". European Early Childhood Education Journal, 20(2), 249-261. doi:10.1080/1350293X.2012.681131

Bessant, K. C. (1995). Factors associated with types of mathematics anxiety in college students. Journal for Research in Mathematics Education, 26(4), 327-345. 
Bieg, M., Goetz, T., Wolter, I., \& Hall, N. C. (2015). Gender stereotype endorsement differentially predicts girls' and boys ' trait-state discrepancy in math anxiety. Frontiers in Psychology, 6(September), 1-8. https://doi.org/10.3389/fpsyg.2015.01404

Blomberg, G., Stürmer, K., \& Seidel, T. (2011). How pre-service teachers observe teaching on video: Effects of viewers' teaching subjects and the subject of the video. Teaching and Teacher Education, 27, 1131-1140.

Blömeke, S. \& Jenßen, L. (2016). A question of validity: Clarifying the hierarchical nature of teacher cognition. In M. Rosén, K. Yang Hansen \& U. Wolff (Eds.), Cognitive abilities and educational achievement: measurement and determinants. A Festschrift in honor of Jan-Eric Gustafsson (pp. 89-110). Cham: Springer International.

Blömeke, S., Jenßen, L., Grassmann, M., Dunekacke, S., \& Wedekind, H. (2017). Process mediates structure: The relation between preschool teacher education and preschool teachers' knowledge. Journal of Educational Psychology, 109(3), 338-354. https://doi.org/10.1037/edu0000147

Blömeke, S., Gustafsson, J.-E., \& Shavelson, R. (2015). Beyond dichotomies: Competence viewed as a continuum. Zeitschrift für Psychologie, 223, 3-13.

Blömeke, S., Jenßen, L., Dunekacke, S., Suhl, U., Grassmann, M., \& Wedekind, H. (2015b). Leistungstests zur Messung der professionellen Kompetenz frühpädagogischer Fachkräfte. Zeitschrift für Pädagogische Psychologie, 29(3-4), 177-191.

Blömeke, S., Kaiser, G., \& Lehmann, R. (2010). TEDS-M 2008. Professionelle Kompetenz und Lerngelegenheiten angehender Primarstufenlehrkräfte im internationalen Vergleich. Münster: Waxmann.

Blömeke, S., König, J., Busse, A., Suhl, U., Benthien, J., Döhrmann, M., \& Kaiser, G. (2014). Von der Lehrerausbildung in den Beruf - Fachbezogenes Wissen als Voraussetzung für Wahrnehmung, Interpretation und Handeln im Unterricht. Zeitschrift für Erziehungswissenschaft, 17, 509-542.

BMFSFJ (Bundesministerium für Familien, Senioren, Frauen und Jugend) (2010). Männliche Fachkräfte in Kindertagesstätten - eine Studie zur Situation von Männern in Kindertagestätten und in der Ausbildung zum Erzieher. Berlin: Sinus Sociovision.

Born-Rauchenecker, E., Drexl, D., \& Weber, K. (2018). Ausbildungsbeispiel: Frühe naturwissenschaftliche Bildung alltagsintegriert umsetzen - ein Unterrichtskonzept. In: Deutsches Jugendinstitut \& Weiterbildungsinitiative Frühpädagogische Fachkräfte (Hrsg.): Frühe naturwissenschaftliche Bildung. Grundlagen für die kompetenzorientierte Weiterbildung. WiFF Wegweiser Weiterbildung, Band 13. München.

Bruns, J. \& Eichen, L. (2018). EmMa - Fortbildung für elementarpädagogische Fachpersonen zur frühen mathematischen Bildung. In: Biehler, R., Lange, T., Leuders, T., RöskenWinter, B., Scherer, P. \& Selter, C. (Hrsg.): Mathematikfortbildungen professionalisieren. (S. 417-434.). Wiesbaden: Springer.

Buckley, S., Reid, K., Goos, M., Lipp, O. V., \& Thomson, S. (2016). Understanding and addressing mathematics anxiety using perspectives from education, psychology and neuroscience. Australian Journal of Education, 60(2), 157-170. https://doi.org/10.1177/0004944116653000

Buxton, L. (1981). Do you panic about maths? Coping with maths anxiety. London

Carey, E., Hill, F., Devine, A., \& Szücs, D. (2016). The Chicken or the Egg? The Direction of the Relationship Between Mathematics Anxiety and Mathematics Performance. Frontiers in Psychology, 6(1987).

Carpenter, T.P., Fennema, E., Peterson, P.L., Chiang, C.-P., \& Loef, M. (1989). Using knowledge of children's mathematics thinking in classroom teaching: an experimental study. American Educational Research Journal, 26(4), 499-531. 
Carey, E., Hill, F., Devine, A., \& Szücs, D. (2016). The chicken or the egg? The direction of the relationship between mathematics anxiety and mathematics performance. Frontiers in Psychology, 6, 1-6. https://doi.org/10.3389/fpsyg.2015.01987

Chang, H. \& Beilock, S. L. (2016). The math anxiety-math performance link and its relation to individual and environmental factors: A review of current behavioral and psychophysiological research. Current Opinion in Behavioral Sciences, 10, 33-38. https://doi.org/10.1016/j.cobeha.2016.04.011

Cherkas, B.M. (1992). A personal essay in math? Getting to know your students. College Teaching, 40(3), 83-86.

Chinn, S. (2012). Beliefs, Anxiety, and Avoiding Failure in Mathematics. Child Development Research, 1-8.

Chipman, S. F., Krantz, D. H., \& Silver, R. (1992). Mathematics anxiety and science careers among able college women. Psychological Science, 3(5), 292-295.

Common Core State Standards Initiative (2014). Common Core State Standards for Mathematics. http://www.corestandards.org/wp-content/uploads/Math_Standards.pdf. Zugegriffen: 1. November 2018.

Cooke, A., Cavanagh, R., Hurst, C. \& Sparrow, L. (2011). Situational effects of mathematics anxiety in pre-service teacher education. Paper presented at the 2011 AARE international Research Conference, Hobart, Australia, 27 November-1 December, 2011. Retrieved from http://www.aare.edu.au/data/publications/2011/aarefinal00501.pdf

Depaepe, F., Verschaffel, L., \& Kelchtermans, G. (2013). Pedagogical content knowledge: A systematic review of the way in which the concept has pervaded mathematics educational research. Teaching and Teacher Education, 34, 12-25.

Dunekacke, S., Jenßen, L., Eilerts, K., \& Blömeke, S. (2016). Epistemological beliefs of prospective pre-school teachers and their relation to knowledge, perception and planning abilities in the field of mathematics: A process-model. ZDM The International Journal on Mathematics Education, 48, 124-137.

Eid, M., Gollwitzer, M., \& Schmitt, M. (2013). Statistik und Forschungsmethoden (3., korrigierte Auflage, 1. Auflage: 2010). Weinheim: Beltz.

Faust, M.W. (1992). Analysis of physiological reactivity in mathematics anxiety. Unveröffentlichte Doktorarbeit, Bowling Green State University, Bowling Green, Ohio.

Franke, M.L., Carpenter, T.P., Levi, L., \& Fennema, E. (2001). Capturing teachers' generative change: a follow-up study of professional development in mathematics. American Educational Research Journal, 38(3), 653-689.

Fröhlich-Gildhoff, K., Nentwig-Gesemann, I., \& Pietsch, S. (2011). Kompetenzorientierung in der Qualifizierung frühpädagogischer Fachkräfte. Eine Expertise der Weiterbildungsinitiative Frühpädagogische Fachkräfte (WiFF). Deutsches Jugendinstitut e.V. München.

Gasteiger, H. \& Benz, C. (2016). Mathematikdidaktische Kompetenz von Fachkräften im Elementarbereich - ein theoriebasiertes Kompetenzmodell. Journal für MathematikDidaktik, 37, 263-287.

Ginet, L., Itzkowich, R., \& Maloney, E. (2018). Math anxiety and math performance: How do they relate? In J.S. McCray, J.-Q. Chen \& J.E. Sorkin (Eds.), Growing mathematical minds. Conversations between developmental psychologists and early childhood teachers (pp. 173-200). New York: Routledge.

Ginsburg, H. P. \& Ertle, B. (2008). Knowing the Mathematics in Early Childhood Mathematics. In O. N. Saracho \& B. Spodek (Hrsg.). Contemporary Perspectives on Mathematics in Early Childhood Education (S. 45-66). Charlotte, NC: Information AGE. 
Goetz, T., Bieg, M., Lüdtke, O., Pekrun, R., \& Hall, N. C. (2013). Do Girls Really Experience More Anxiety in Mathematics? Psychological Science, 24(10), 2079-2087. https://doi.org/10.1177/0956797613486989

Goldstein, E.B. (2002). Wahrnehmungspsychologie. Heidelberg: Spektrum.

Gresham, G. (2007). A Study of Mathematics Anxiety in Pre-Service Teachers. Early Childhood Education Journal, 35(2), 181-188.

Gresham, G. (2018). Preservice to Inservice: Does Mathematics Anxiety Change With Teaching Experience? Journal of Teacher Education, 69(1), 90-107. https://doi.org/10.1177/0022487117702580

Gresham, G. \& Burleigh, C. (2018). Exploring early childhood preservice teachers' mathematics anxiety and mathematics efficacy beliefs. Teaching Education, 1-25. https://doi.org/10.1080/10476210.2018.1466875

Gudjons, H. (2008). Handlungsorientiert lehren und lernen: Schüleraktivierung Selbsttätigkeit - Projektarbeit. 7., aktualisierte Auflage. Bad Heilbrunn: Klinkhardt.

Hembree, R. (1990). The Nature, Effects, and Relief of Mathematics Anxiety. Journal for Research in Mathematics Education, 21(1), 33-46.

Hill, F., Mammarella, I. C., Devine, A., Caviola, S., Passolunghi, M. C., \& Szucs, D. (2016). Maths anxiety in primary and secondary school students: Gender differences, developmental changes and anxiety specificity. Learning and Individual Differences, 48, 45-53. https://doi.org/10.1016/j.lindif.2016.02.006

Hill, H. C., Rowan, B., \& Ball, D. L. (2005). Effects of teachers' mathematical knowledge for teaching on student achievement. American Educational Research Journal, 42(2), 371406.

Hoorfar, H. \& Taleb, Z. (2015). Correlation Between Mathematics Anxiety with Metacognitive Knowledge. Procedia - Social and Behavioral Sciences, 182, 737-741. https://doi.org/10.1016/j.sbspro.2015.04.822

Hunt, T. E., Clark-Carter, D., \& Sheffield, D. (2014). Math anxiety, intrusive thoughts and performance. Journal of Education, Psychology and Social Sciences, 2(2), 69-75.

Hunt, T., Clark-Carter, D., \& Sheffield, D. (2015). Exploring the Relationship Between Mathematics Anxiety and Performance: An Eye-Tracking Approach. Applied Cognitive Psychology, 29, 226-231.

Jansen, B. R. J., Louwerse, J., Straatemeier, M., Van der Ven, S. H. G., Klinkenberg, S., and Van der Maas, H. L. J. (2013). The influence of experiencing success in math on math anxiety, perceived math competence, and math performance. Learning and individual differences, 24, 190-197. doi: 10.1016/j.lindif.2012.12.014

Jansen, B. R. J., Schmitz, E. A., \& van der Maas, H. L. J. (2016). Affective and motivational factors mediate the relation between math skills and use of math in everyday life. Frontiers in Psychology, 7(APR), 1-11. https://doi.org/10.3389/fpsyg.2016.00513

Jenßen, L. (2017). "Mathe in der Kita? Gibt's doch gar nicht!" Validierung eines Tests zur Erfassung des mathematischen Fachwissens angehender Erzieherinnen. Dissertation. Berlin: Humboldt-Universität zu Berlin.

Jenßen, L., Dunekacke, S., \& Blömeke, S. (2015). Qualitätssicherung in der Kompetenzforschung. Kompetenzen von Studierenden. 61. Beiheft Der Zeitschrift Für Pädagogik, 2015, 11-31.

Jenßen, L., Dunekacke, S., Baack, W., Tengler, M., Koinzer, T., Schmude, C., Grassmann, M., Wedekind, H., \& Blömeke, S. (2015). KomMa: Kompetenzmodellierung und Kompetenzmessung bei frühpädagogischen Fachkräften im Bereich Mathematik. In B. Koch-Priewe, A. Köker, J. Seifried \& E. Wuttke (Hrsg.), Kompetenzerwerb an Hochschulen: Modellierung und Messung. Zur Professionalisierung angehender Lehrerinnen und Lehrer sowie frühpädagogischer Fachkräfte (S.59-80). Bad Heilbrunn: Verlag Julius Klinkhardt. 
Jenßen, L., Dunekacke, S., Eid, M., \& Blömeke, S. (2015). The relationship of mathematical competence and mathematics anxiety: An application of latent state-trait theory. Zeitschrift für Psychologie, 223(1), 31-38.

Kahan, J. A., Cooper, D. A., \& Bethea, K. A. (2003). The role of mathematics teachers' content knowledge in their teaching: a framework for research applied to a study of student teachers. Journal of Mathematics Teacher Education, 6, 223-252.

Kaiser, G., Blömeke, S., König, J., Busse, A., Döhrmann, M., \& Hoth, J. (2017). Professional competencies of (prospective) mathematics teachers - cognitive versus situated approach. Educational Studies in Mathematics, 94, 161-182.

Karp, K. S. (1991). Elementary School Teachers' Attitudes Toward Mathematics: The Impact on Students' Autonomous Learning Skills. School Science and Mathematics, 91(6), 265270, doi:10.1111/j.1949-8594.1991.tb12095.x.

Kelly, W.P. \& Tomhave, W.K. (1985). A Study of Math Anxiety/Math Avoidance in Preservice Elementary Teachers. The Arithmetic Teacher, 32(5), 51-53.

Kersting, N. B., Givvin, K., Sotelo, F., \& Stigler, J. W. (2010). Teacher's analysis of classroom video predicts student learning of mathematics: Further exploration of a novel measure of teacher knowledge. Journal of Teacher Education, 61, 172-181.

Kleeberger, F., \& Stadler, K. (2011). Zehn Fragen - zehn Antworten: Die Ausbildung von Erzieherinnen und Erziehern aus Sicht der Lehrkräfte: Ergebnisse einer bundesweiten Befragung von Lehrkräften an Fachschulen für Sozialpädagogik: eine Studie der Weiterbildungsinitiative Frühpädagogische Fachkräfte (WiFF). WiFF-Studien Ausbildung: Vol. 13. München: DJI.

Klein, F. (1908): Elementarmathematik vom höheren Standpunkte aus. Arithmetik, Algebra, Analysis. Berlin: Julius Springer.

Klibanoff, R.S., Levine, S.C., Huttenlocher, J., Vasilyeva, M., \& Hedges, L.V. (2006). Preschool children's mathematical knowledge: The effect of teacher "math talk". Developmental Psychology, 42(1), S. 59-69.

KMK. (2017). Kompetenzorientiertes Qualifikationsprofil für die Ausbildung von Erzieherinnen und Erziehern an Fachschulen und Fachakademien: Beschluss der Kultusministerkonferenz vom 01.12.2011 i.d.F. vom 24.11.2017.

Knievel, I., Lindmeier, A. M., \& Heinze, A. (2015). Beyond knowledge: Measuring primary teachers' subject-specific competences in and for teaching mathematics with items based on video vignettes. International Journal of Science and Mathematics Education, 13(2), 309-329.

König, J. \& Lebens, M. (2012). Classroom Management Expertise (CME) von Lehrkräften messen: Überlegungen zur Testung mithilfe von Videovignetten und erste empirische Befunde. Lehrerbildung auf dem Prüfstand, 5(1), 3-29.

König, J., Blömeke, S., Klein, P., Suhl, U., Busse, A., \& Kaiser, G. (2014). Is teachers' general pedagogical knowledge a premise for noticing and interpreting classroom situations? A video-based assessment approach. Teaching and Teacher Education, 38, 76-88.

Lee, J., Meadows, M., \& Lee, J. O. (2003). What causes teachers to implement high quality mathematics education more frequently: focusing on teachers' pedagogical content knowledge. Washington: ERIC.

Liebig, M. (2016). Die Fachschulausbildung zur Erzieherin/zum Erzieher unter Druck: Entwicklungslinien in der Ausbildung frühpädagogischen Fachpersonals. bwp@ Berufs- Und Wirtschaftspädagogik - Online, 31, 1-20.

Lindmeier, A., Hepberger, B., Heinze, A., \& Moser Opitz, E. (2016). Modeling cognitive dispositions of educators for early mathematics education. Proceedings of the 40th Conference of the International Group for the Psychology of Mathematics Education, 3, 219-226. 
Little, T. D., Cunningham, W. A., Shahar, G., \& Widaman, K. F. (2002) To parcel or not to parcel: Exploring the question, weighing the merits, Structural Equation Modeling: A Multidisciplinary Journal, 9(2), 151-173.

Lord, F.M. \& Novick, M.R. (1968). Statistical theories of mental test scores. Reading: AddisonWesley.

Ma, X. (1999). A Meta-Analysis of the Relationship between Anxiety toward Mathematics and Achievement in Mathematics. Journal for Research in Mathematics Education, 30(5), 520-540.

Maloney, E. A., \& Beilock, S. L. (2012). Math anxiety: Who has it, why it develops, and how to guard against it. Trends in Cognitive Sciences, 16(8), 404-406.

McCray, J. S., \& Chen, J.-Q. (2012). Pedagogical Content Knowledge for Preschool Mathematics: Construct Validity of a New Teacher Interview. Journal of Research in Childhood Education, 26(3), 291-307.

Miller, H. \& Bichsel, J. (2004). Anxiety, working memory, gender, and math performance. Personality and Individual Differences, 37, 591-606.

Ministerium für Kultus, Jugend und Sport Baden-Württemberg (2011). Orientierungsplan für Bildung und Erziehung in baden-württembergischen Kindergärten und weiteren Kindertageseinrichtungen.

Mizala, A., Martínez, F., \& Martínez, S. (2015). Pre-service elementary school teachers' expectations about student performance: How their beliefs are affected by their mathematics anxiety and student's gender. Teaching and Teacher Education, 50, 7078. https://doi.org/10.1016/j.tate.2015.04.006

Morawiak, U., Schulz, A., Jungmann, T., \& Koch, K. (2017). Professionalisierungsangebote im KOMPASS-Projekt. In T. Jungmann \& K. Koch (Hrsg.). Professionalisierung pädagogischer Fachkräfte in Kindertageseinrichtungen (S. 29-64). Wiesbaden: Springer.

Muthén, L. K. \& Muthén, B. O. (2017). Mplus User's Guide. Eighth Edition. Los Angeles: Muthén \& Muthén.

Pekrun, R. (2006). The control-value theory of achievement emotions: Assumptions, corollaries, and implications for educational research and practice. Educational Psychology Review, 18(4), 315-341.

Pekrun, R., Muis, K.R., Frenzel, A.C., \& Goetz, T. (2017). Emotions at school. New York, US: Routledge.

Pekrun, R. \& Linnenbrink-Garcia,L. (2014). International handbook of emotions in education. New York, US: Routledge.

Pizzie, R. G. \& Kraemer, D. J. M. (2017). Avoiding math on a rapid timescale: Emotional responsivity and anxious attention in math anxiety. Brain and Cognition, 118(March), 100-107. https://doi.org/10.1016/j.bandc.2017.08.004

Pólya, G. (1977). Mathematical Methods in Science. Washington, DC: Mathematical Association of America.

Porsch, R. (2018). Emotionen in der LehrerInnenbildung. In M. Huber \& S. Krause, (Hrsg.), Bildung und Emotion (S. 269-287). Wiesbaden: Springer VS.

Porsch, R. (2019). Mathematikangst bei angehenden Lehrkräften - Ein systematisches Review internationaler Forschungsarbeiten. Mathematica Didatica, 42, 1-24.

Porsch, R., Strietholt, R., Macharski, T., \& Bromme, R. (2015). Mathematikangst im Kontext - Ein Inventar zur situationsbezogenen Messung von Mathematikangst bei angehenden Lehrkräften. Journal für Mathematik-Didaktik, 36(1), 1-22. https://doi.org/10.1007/s13138-014-0067-4

Ramirez, G., Shaw, S. T., \& Maloney, E. A. (2018). Math Anxiety: Past Research, Promising Interventions, and a New Interpretation Framework. Educational Psychologist, 1520, 1-20. https://doi.org/10.1080/00461520.2018.1447384 
Richardson, F. \& Suinn, R. (1972). The mathematics anxiety rating scale; Psychometric Data. Journal of Counseling Psychology, 19(6), 551-554.

Rosemann, B. \& Kerres, M. (1985). Bedingungen des Lehrerverhaltens: Rationale, emotionale und zirkuläre Prozesse bei der Situationswahrnehmung. Psychologie in Erziehung und Unterricht, 32(4), 241-247.

Rost (2008). Zur Messung von Kompetenzen einer Bildung für nachhaltige Entwicklung. In I. Bormann \& G. De Haan (Hrsg.), Kompetenzen der Bildung für nachhaltige Entwicklung. Operationalisierung, Messung, Rahmenbedingung, Befunde (S. 61-73). Wiesbaden: VS Verlag für Sozialwissenschaften.

Santagata, R. \& Guarino, J. (2011). Using video to teach future teachers to learn from teaching. ZDM Mathematics Education, 43,133-145.

Santagata, R. \& Yeh, C. (2013). Learning to teach mathematics and to analyze teaching effectiveness: Evidence from a video- and practice-based approach. Journal of Mathematics Teacher Education, 17(6), 491-514.

Santagata, R., \& Yeh, C. (2016). The role of perception, interpretation, and decision making in the development of beginning teachers' competence. ZDM, 48(1-2), 153-165.

Schoenfeld, A. H. \& Kilpatrick, J. (2008). Toward a theory of proficiency in teaching mathematics. In D. Tirosh \& T. Woods (Eds.), The international handbook of mathematics teacher education (pp. 321-354). Rotterdam: Sense Publishers.

Schukajlow, S., Rakoczy, K., \& Pekrun, R. (2017). Emotions and motivation in mathematics education: theoretical considerations and empirical contributions. ZDM - Mathematics Education, 49(3), 307-322. https://doi.org/10.1007/s11858-017-0864-6

Schuler, S., Streit, C., \& Wittmann, G. (2017). Perspektiven mathematischer Bildung im Übergang vom Kindergarten zur Grundschule. Wiesbaden: Springer Spektrum.

Schuler, S, Wittmann, G., Levin, A., \& Bönig, D. (2017). Das intendierte Handeln in offenen Lehr-Lern-Situationen als Indikator für die mathematikbezogene Kompetenz von ErzieherInnen und GrundschullehrerInnen. In S. Schuler, C. Streit, \& G. Wittmann (Hrsg.): Perspektiven mathematischer Bildung im Übergang vom Kindergarten zur Grundschule (S. 223-237). Wiesbaden: Spektrum.

Schweer, M.K.W. (1992). Handeln in sozialen Berufen. Essen: Plöger Verlag.

Schweer, M.K.W. \& Thies, B. (2000) Situationswahrnehmung und interpersonales Verhalten im Klassenzimmer. In M.K.W. Schweer (Hrsg.). Lehrer-Schüler-Interaktion (S. 59 78). Wiesbaden: Springer.

Seidel, T. \& Prenzel, M. (2007). Wie Lehrpersonen Unterricht wahrnehmen und einschätzen Erfassung pädagogisch-psychologischer Kompetenzen mit Videosequenzen. In M. Prenzel, I. Gogolin \& H.-H. Krüger (Hrsg.). Kompetenzdiagnostik (S. 201-216). Wiesbaden: VS Verlag (Sonderheft, 8).

Sherin, M.G. (2007). The development of teachers' professional vision in video clubs. In R. Goldman, R. Pea, B. Barron \& S.J. Derry (Hrsg.), Video research in learning sciences (S. 383-395). Mahwah: Lawrence Erlbaum.

Sokolowski, H. M., Hawes, Z., \& Lyons, I. M. (2019). What explains sex differences in math anxiety? A closer look at the role of spatial processing. Cognition, 182, 193-212. https://doi.org/10.1017/CBO9781107415324.004

Stahnke, R., Schüler, S., \& Rösken-Winter (2016). Teachers' perception, interpretation, and decision- making: a systematic review of empirical mathematics education research. ZDM The International Journal on Mathematics Education, 48, 1-27. doi: $10.1007 / \mathrm{s} 11858-016-0775-\mathrm{y}$

Star, J. R. \& Strickland, S. K. (2008). Learning to observe: Using video to improve preservice mathematics teachers' ability to notice. Journal for Mathematics Teacher Education, 11(2), $107-125$. 
Stoehr, K. J. (2017). Building the wall brick by brick: one prospective teacher's experiences with mathematics anxiety. Journal of Mathematics Teacher Education, 20(2), 119-139. https://doi.org/10.1007/s10857-015-9322-y

Thiel, O. (2010). Teachers' attitudes towards mathematics in early childhood education. European Early Childhood Education Research Journal, 18(1), 105-115. doi: $10.1080 / 13502930903520090$

Thiel, O. \& Jenßen, L. (2018). Affective-motivational aspects of early childhood teacher students' knowledge about mathematics. European Early Childhood Education Research Journal, 26(4), 512-534. https://doi.org/10.1080/1350293X.2018.1488398

Thonhauser, J. (2007). Lehrer/-innen handeln situationsspezifisch. In A. Gastager, T. Hascher \& H. Schwetz (Hrsg.), Pädagogisches Handeln: Balance zwischen Theorie und Praxis. Beiträge zur Wirksamkeitsforschung in pädagogisch-psychologischem Kontext. Erziehungswissenschaft, Bd. 24 (S. 47 - 60). Landau: VEP.

Tsamir, P., Tirosh, D., Levenson, E., Tabach, M., \& Barkai, R. (2014). Employing the CAMTE framework: Focusing on preschool teachers' knowledge and self-efficacy related to students' conceptions. In U. Kortenkamp, B. Brandt, C. Benz, G. Krummheuer, S. Ladel \& R. Vogel (Eds.), Early Mathematics Learning (pp. 291-306). New York: Springer Science+Business.

van Es, E.A. \& Sherin, M.G. (2008). Mathematics teachers' "learning to notice" in the context of a video club. Teaching and Teacher Education, 24, 244-276.

Wittmann, Böning, Levin \& Schuler (2016). Computergestützte Erhebung: Ergebnisse. In G. Wittmann, A. Levin, \& D. Böning (Hrsg.), AnschlussM. Anschlussfähigkeit mathematikdidaktischer Überzeugungen und Praktiken von ErzieherInnen und GrundschullehrerInnen (S. 200-217). Wiesbaden: Springer.

Young, C. B., Wu, S. S., \& Menon, V. (2012). The Neurodevelopmental Basis of Math Anxiety. Psychological Science, 23(5), 492-501. https://doi.org/10.1177/0956797611429134 